University of North Florida

UNIVERSITY of

NORTH FLORIDA.

UNF Digital Commons

$12-2001$

\title{
Prediction of the Crystal Structures of Perovskites Using the Software Program SPuDS
}

Michael W. Lufaso

University of North Florida, michael.lufaso@unf.edu

Patrick M. Woodward

Follow this and additional works at: https://digitalcommons.unf.edu/achm_facpub

Part of the Chemistry Commons

\section{Recommended Citation}

Lufaso, Michael W. and Woodward, Patrick M., "Prediction of the Crystal Structures of Perovskites Using the Software Program SPuDS" (2001). Chemistry Faculty Publications. 4.

https://digitalcommons.unf.edu/achm_facpub/4

This Article is brought to you for free and open access by the Department of Chemistry at UNF Digital Commons. It has been accepted for inclusion in Chemistry Faculty Publications by an authorized administrator of UNF Digital Commons. For more information, please contact Digital Projects.

(C) 12-2001 All Rights Reserved

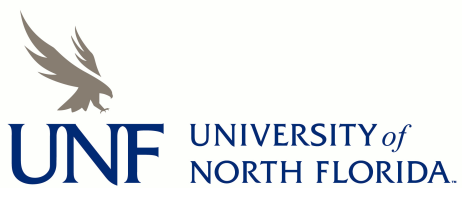


Acta Crystallographica Section B

Structural

Science

ISSN 0108-7681

\section{Michael W. Lufaso and Patrick M. Woodward*}

100 West 18th Avenue, Columbus, OH 43210, USA

Correspondence e-mail: woodward@chemistry.ohio-state.edu

\section{Prediction of the crystal structures of perovskites using the software program SPuDS}

The software program SPuDS has been developed to predict the crystal structures of perovskites, including those distorted by tilting of the octahedra. The user inputs the composition and SPUDS calculates the optimal structure in ten different Glazer tilt systems. This is performed by distorting the structure to minimize the global instability index, while maintaining rigid octahedra. The location of the $A$-site cation is chosen so as to maximize the symmetry of its coordination environment. In its current form $S P u D S$ can handle up to four different $A$-site cations in the same structure, but only one octahedral ion. Structures predicted by $S P U D S$ are compared with a number of previously determined structures to illustrate the accuracy of this approach. SPuDS is also used to examine the prospects for synthesizing new compounds in tilt systems with multiple $A$-site coordination geometries $\left(a^{+} a^{+} a^{+}, a^{0} b^{+} b^{+}, a^{0} b^{-} c^{+}\right)$.

\section{Introduction}

The perovskite structure type is one of the most frequently encountered in solid-state inorganic chemistry. The ideal perovskite structure has $A B X_{3}$ stoichiometry and is composed of a three-dimensional framework of corner-sharing $B X_{6}$ octahedra. The $A$-site cation fills the 12 coordinate cavities formed by the $B X_{3}$ network and is surrounded by 12 equidistant anions. The perovskite structure accommodates most of the metallic ions in the periodic table and a significant number of different anions. The majority of the perovskite compounds are oxides or fluorides, but the perovskite structure is also known for the heavier halides (Hönle et al., 1988; Luaña et al., 1997), sulfides (Clearfield, 1963), hydrides (Gingl et al., 1999), cyanides (Peschel et al., 2000; Malecki \& Ratuszna, 1999), oxyfluorides (Carlson et al., 2000) and oxynitrides (Marchand et al., 1991). The physical properties of perovskite materials are equally diverse. Perovskites with ferroelectric and/or piezoelectric properties, such as $\mathrm{BaTiO}_{3}, \mathrm{~Pb}\left(\mathrm{Zr}_{1-x} \mathrm{Ti}_{x}\right) \mathrm{O}_{3}$ and $\mathrm{Pb}_{2} \mathrm{ScTaO}_{6}$, play a dominant role in the electroceramics industry. Such materials also serve as critical components in a number of smart devices (Newnham, 1997; Trolier-McKinstry \& Newnham, 1993). Doped $\mathrm{BaBiO}_{3}$ (Sleight et al., 1975) has the highest superconducting transition temperature for a noncuprate oxide material, not to mention the fact that the hightemperature cuprate superconductors adopt perovskiterelated crystal structures. Members of the manganate-based perovskite system, $\left(\operatorname{Ln}_{1-x} A_{x}\right) \mathrm{MnO}_{3}(\mathrm{Ln}=$ lanthanide ion, $A=$ alkaline earth ion), have been studied extensively over the past decade for their colossal magnetoresistive (CMR) properties.
Received 9 March 2001

Accepted 18 September 2001
(C) 2001 International Union of Crystallography Printed in Great Britain - all rights reserved 
38 Interestingly, the mineral perovskite, $\mathrm{CaTiO}_{3}$, does not adopt the aristotype cubic structure. The symmetry of $\mathrm{CaTiO}_{3}$ is lowered from cubic $(P m \overline{3} m, Z=1)$ to orthorhombic (Pnma, $Z=4$ ) by a cooperative tilting of the titanium-centered octahedra (Sasaki et al., 1987). This distortion is driven by the mismatch between the size of the cubo-octahedral cavity in the corner-sharing octahedral network and the undersized ionic radius of the $\mathrm{Ca}^{2+}$ ion. The octahedral tilting distortion lowers the coordination number of $\mathrm{Ca}^{2+}$ from 12 to 8 , reduces the tension in the remaining $\mathrm{Ca}-\mathrm{O}$ bonds (Brown, 1992) and increases the lattice energy. However, there is very little perturbation of the local octahedral coordination of the $\mathrm{Ti}^{4+}$ ion. It is fitting that the mineral perovskite adopts a distorted structure, since distorted perovskites far outnumber undistorted cubic perovskites (Fig. 1). In fact, the prevalence of the perovskite structure type can be directly attributed to the inherent ability of the corner-sharing octahedral framework to undergo cooperative octahedral tilting distortions in response to the size mismatch between the $A$ and $B$ cations.

The presence and magnitude of an octahedral tilting distortion affects not only the crystal structure, but also has a profound influence on a number of physical properties, such as electrical conductivity, magnetic superexchange interactions and certain dielectric properties. For example, $\mathrm{Ln}_{0.7} A_{0.3} \mathrm{MnO}_{3}$ perovskites undergo a transition from a paramagnetic insulating state to a ferromagnetic metallic state upon cooling. This coupled electronic/magnetic transition is of great interest due to the fact that the magnetoresistance reaches a maximum value as the temperature approaches this transition. Furthermore, it is known that the transition temperature can be tuned from $\sim 350 \mathrm{~K}$ to below $100 \mathrm{~K}$ by changing the magnitude of the octahedral tilting (Hwang et al., 1995). This remarkable sensitivity to a relatively subtle structural distortion originates from the decrease in orbital overlap that occurs as the octahedral tilting distortion increases (Töpfer \& Goodenough, 1997). Another example of coupling between the octahedral tilting distortion and a physical property of technological significance occurs in perovskites used for microwave dielectric applications. Colla et al. (1993) have shown that the sign

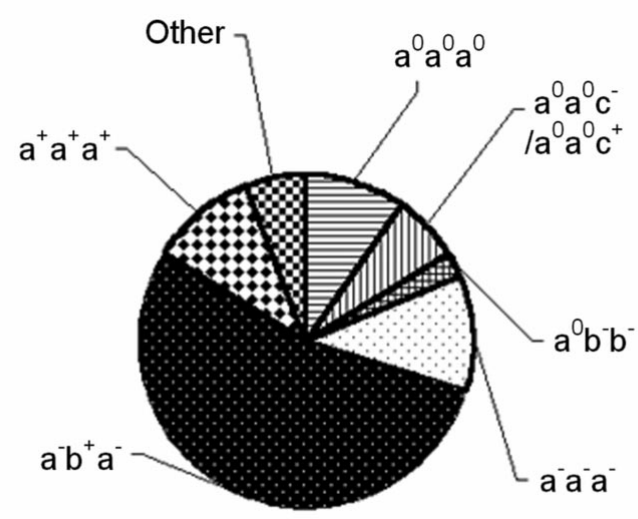

\section{Figure 1}

Distribution of tilt systems among known perovskites with a single octahedral cation. and magnitude of the temperature coefficient of the dielectric constant is quite sensitive to changes in the octahedral tilting distortion.

The prevalence and importance of octahedral tilting distortions provide clear motivation to develop software capable of predicting distorted perovskite crystal structures. One step toward this goal was the development of the program POTATO (Woodward, 1997a), which was used in the high-pressure, high-temperature synthesis of two new perovskites containing monovalent silver (Park et al., 1998). Unfortunately, POTATO cannot easily be used for structure prediction because the required input data (the $B-X$ bond distance, the octahedral tilt system and the magnitude of the tilting distortion) are not known in advance of synthesis and structural characterization. This shortcoming motivated our efforts to develop a more advanced software package capable of predicting perovskite crystal structures directly from the composition. The fruit of this labor is a new software package entitled SPuDS (Structure Prediction Diagnostic Software), which is described and evaluated in this study. We anticipate that SPUDS will find a number of useful applications, including:

(i) Predicted structures can be used to estimate physical (magnetic, dielectric and other) properties of both hypothetical compositions and those materials for which accurate structural data is not available.

(ii) $S P U D S$ can be used as a guide for exploratory synthetic efforts. It should be particularly useful for compounds with multiple cations on the $A$-site (i.e. $\mathrm{CaCu}_{3} \mathrm{Ti}_{4} \mathrm{O}_{12}$ ), as well as high-pressure synthesis, where access to experimental facilities is limited.

(iii) Predicted structures can serve as the starting point for Rietveld refinements in the course of structurally characterizing new materials.

(iv) Structures generated by SPuDS can be compared with experimentally determined structures in order to deconvolute the effects of octahedral tilting distortions from other structural distortion mechanisms.

\section{Octahedral tilt systems: description and notation}

As previously noted, octahedral tilting reduces the symmetry of the $A$-site cation coordination environment and results in a change in $A-X$ bond lengths. However, there are multiple ways in which the octahedra can tilt, each leading to a different coordination environment for the $A$-site cation(s). The coordination number and geometry of the first coordination sphere has been described previously for some common tilt systems (Woodward, 1997b).

A standard notation has been developed to describe octahedral tilting distortions in perovskites (Glazer, 1972). An alternative, but equally valid, notation was developed by Aleksandrov (1976). For the sake of clarity we will use the Glazer notation throughout this paper. The notation describes a tilt system by rotations of $B X_{6}$ octahedra about the three orthogonal Cartesian axes, which are coincident with the three 
Table 1

The 15 tilt systems, space groups, degrees of freedom, number of independent Wyckoff sites and number of observed structures reported for simple perovskites with the restriction that not more than two layers show independent tilting.

For the Glazer tilt system the number in parentheses corresponds to the numbering of the tilt systems originally adopted by Glazer (1972).

\begin{tabular}{|c|c|c|c|c|c|c|}
\hline \multirow[b]{2}{*}{ Glazer tilt system } & \multirow[b]{2}{*}{ Space group } & \multirow[b]{2}{*}{ Degrees of freedom } & \multicolumn{3}{|c|}{ Wyckoff sites } & \multirow[b]{2}{*}{ Frequency } \\
\hline & & & $A$ & $B$ & $X$ & \\
\hline \multicolumn{7}{|c|}{ Group A: High-symmetry tilt systems } \\
\hline$a^{0} a^{0} a^{0}(23)$ & $\operatorname{Pm} \overline{3} m$ & 1 & 1 & 1 & 1 & 21 \\
\hline$a^{-} a^{-} a^{-}(14)$ & $R \overline{3} c$ & 3 & 1 & 1 & 1 & 24 \\
\hline$a^{0} a^{0} c^{-}(22)$ & $I 4 / \mathrm{mcm}$ & 3 & 1 & 1 & 2 & 9 \\
\hline$a^{0} a^{0} c^{+}(21)$ & $P 4 / m b m$ & 3 & 1 & 1 & 2 & 5 \\
\hline$a^{0} b^{-} b^{-}(20)$ & Imma & 6 & 1 & 1 & 2 & 6 \\
\hline$a^{-} b^{+} a^{-}(10)$ & Pnma & 10 & 1 & 1 & 2 & 119 \\
\hline \multicolumn{7}{|c|}{ Group B: Multiple $A$-site tilt systems } \\
\hline$a^{+} a^{+} a^{+}(3)$ & $\operatorname{Im} \overline{3}$ & 3 & 2 & 1 & 1 & 22 \\
\hline$a^{0} b^{-} c^{+}(17)$ & $\mathrm{Cmcm}$ & 10 & 2 & 1 & 3 & 6 \\
\hline$a^{0} b^{+} b^{+}(16)$ & $I 4 / \mathrm{mmm}$ & 5 & 3 & 1 & 2 & 0 \\
\hline$a^{+} a^{+} c^{-}(5)$ & $P 4_{2} / n m c$ & 8 & 3 & 1 & 3 & 1 \\
\hline \multicolumn{7}{|c|}{ Group C - Transitional/low-symmetry tilt systems } \\
\hline$a^{-} a^{-} c^{-}(13)$ & $C 2 / c$ & 9 & 1 & 1 & 2 & 0 \\
\hline$a^{0} b^{-} c^{-}(19)$ & $C 2 / m$ & 10 & 1 & 1 & 3 & 2 \\
\hline$a^{-} b^{-} c^{-}(12)$ & $P \overline{1}$ & 18 & 1 & 2 & 3 & 1 \\
\hline$a^{+} b^{-} c^{-}(8)$ & $P 2_{1} / m$ & 18 & 2 & 2 & 4 & 3 \\
\hline$a^{+} b^{+} c^{+}(1)$ & Immm & 9 & 4 & 1 & 3 & 0 \\
\hline
\end{tabular}

axes of the aristotype cubic unit cell. The letters in Glazer's notation indicate the relative magnitude of the rotation about a given axis, e.g. use of the letters $a, b$ and $c$ imply unequal tilts about the $x, y$ and $z$ axes. A superscript is used to denote the phase of the octahedral tilting in neighboring layers. A positive superscript would denote the neighboring octahedra tilt in the same direction (in-phase) and a negative superscript implies the tilts of neighboring octahedra tilt in the opposite direction (out of phase). Superscript 0 signifies no tilting about that axis. Fig. 2 illustrates the structures which correspond to tilt systems $a^{0} a^{0} c^{+}$and $a^{0} a^{0} c^{-}$. The octahedral rotations in tilt systems $a^{0} a^{0} c^{+}$and $a^{0} a^{0} c^{-}$occur only about the $z$ axis of the cubic perovskite. Rotation of one octahedron causes the four adjacent octahedra in the same layer to rotate in the opposite direction by the same amount. From this figure one can see that

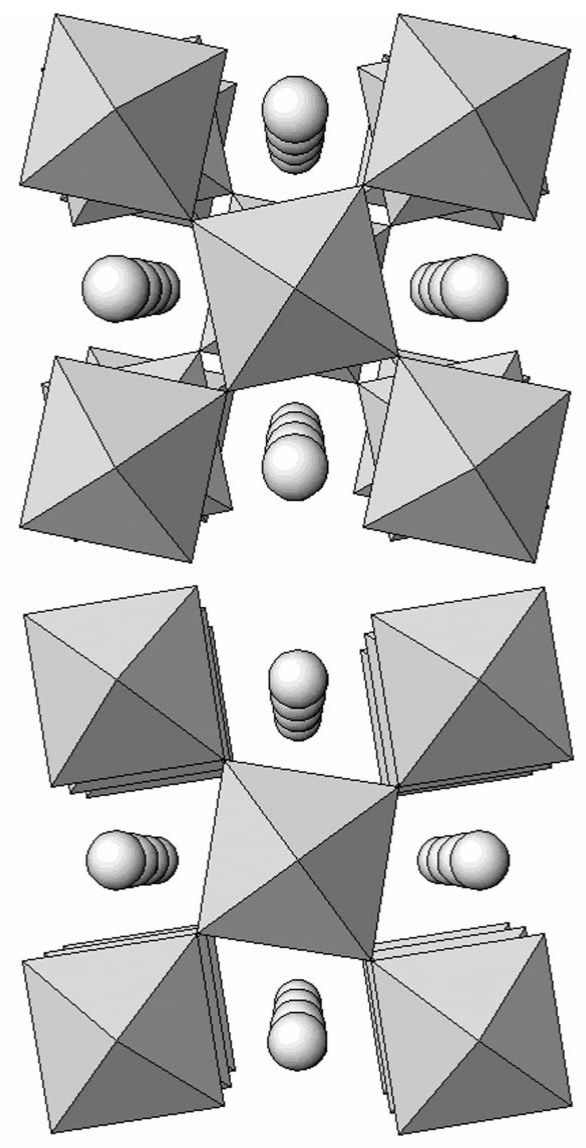

Figure 2

View looking down the $c$ axis of $a^{0} a^{0} c^{-}$(top) and $a^{0} a^{0} c^{+}$(bottom) with the $A$-site cations shown as spheres and the $B$-site cations located at the center of the octahedra. rotation of a single octahedron defines the rotation of all such that rotations of the octahedra in the layer above and below are not geometrically constrained to the initial rotation and can occur in phase (+ superscript) or out of phase (superscript) with respect to the first octahedral layer.

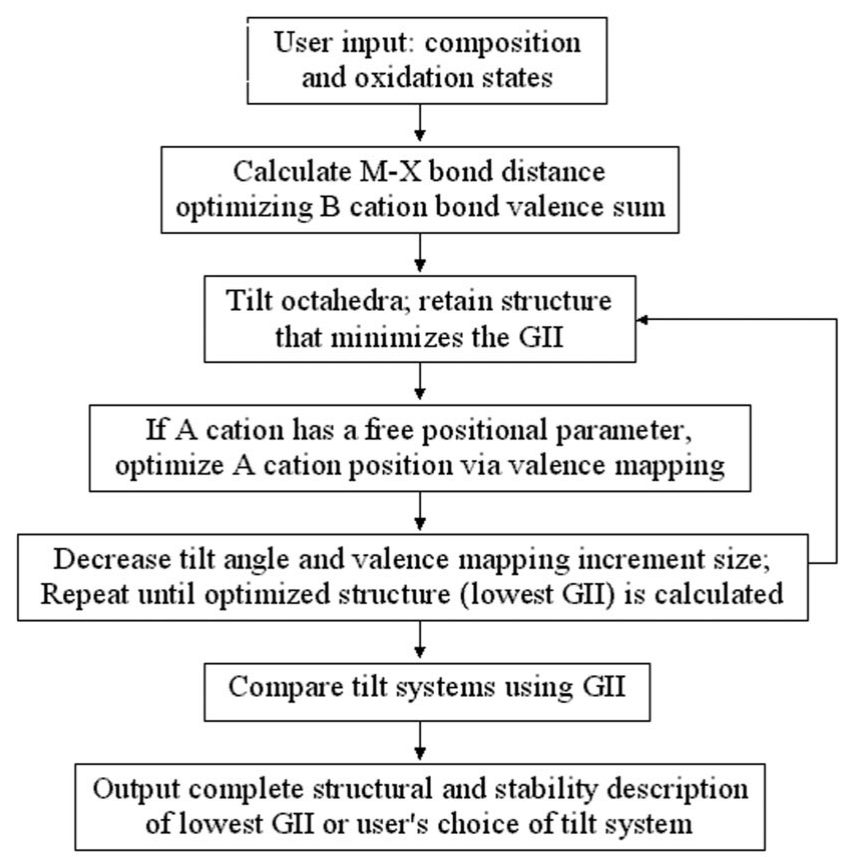

Figure 3

SPUDS operational flowchart. octahedra in the same layer. However, lattice connectivity is 
Table 2

Lattice parameter equations based on $B-X$ bond distance $(d)$ and tilt angle.

The angle $\varphi$ is the octahedral tilt about the cubic [001], $\theta$ is the octahedral tilt angle about the cubic [110], $\Phi$ is the octahedral angle about the cubic [111] and $\omega$ is the octahedral tilt angle about the cubic [011].

\begin{tabular}{|c|c|c|}
\hline Glazer tilt & Space group & $\begin{array}{l}\text { Lattice parameters } \\
(d=B-X \text { bond distance })\end{array}$ \\
\hline $\begin{array}{l}a^{0} a^{0} a^{0}(23) \\
a^{0} a^{0} c^{-}(22)\end{array}$ & $\begin{array}{l}P m \overline{3} m \\
I 4 / m c m\end{array}$ & $\begin{array}{l}a=2 d \\
a=8^{1 / 2} d \cos \varphi \\
c=4 d\end{array}$ \\
\hline$a^{0} a^{0} c^{+}(21)$ & $P 4 / \mathrm{mbm}$ & $\begin{array}{l}c=4 d \\
a=8^{1 / 2} d \cos \varphi \\
c=2 d\end{array}$ \\
\hline$a^{0} b^{-} b^{-}(20)$ & Imma & $\begin{array}{l}a=8^{1 / 2} d \\
b=4 d \cos \theta \\
c=8^{1 / 2} d \cos \theta\end{array}$ \\
\hline$a^{0} b^{-} c^{+}(17)$ & $\mathrm{Cmcm}$ & $\begin{array}{l}a=4 d \cos \theta \\
b=2 d(\cos \theta+1) \\
c=2 d(\cos \theta+1)\end{array}$ \\
\hline$a^{0} b^{+} b^{+}(16)$ & $I 4 / \mathrm{mmm}$ & $\begin{array}{l}a=2 d(1+\cos \theta) \\
c=4 d \cos \theta\end{array}$ \\
\hline$a^{-} a^{-} a^{-}$(14) & $R \overline{3} c$ & $\begin{array}{l}a=8^{1 / 2} d \cos \Phi \\
c=(48)^{1 / 2} d\end{array}$ \\
\hline$a^{+} b^{-} b^{-}(10)$ & Pnma & $\begin{array}{l}a=d\left[8\left(2+\cos ^{2} \omega / 3\right)\right]^{1 / 2} \\
b=d\left[48 /\left(1+2 \sec ^{2} \omega\right)\right]^{1 / 2} \\
c=8^{1 / 2} d \cos \omega\end{array}$ \\
\hline & $P 4_{2} / n m c$ & $\begin{array}{l}a=2 d\{\cos \varphi+\sin \varphi-\cos \theta[\sin \varphi-\cos \varphi]\} \\
c=4 d \cos \theta\end{array}$ \\
\hline$a^{+} a^{+} a^{+}$(3) & $\operatorname{Im} \overline{3}$ & $a=d(8 \cos \Phi+4) / 3$ \\
\hline
\end{tabular}

38 The tilting of the octahedra reduces the symmetry of the undistorted perovskite tilt system $a^{0} a^{0} a^{0}$. Glazer derived 23 different tilt systems, which led to 15 different space groups (Glazer, 1972). Minor corrections to the space groups were published in updated descriptions (Glazer, 1975; Burns \& Glazer, 1990). Howard \& Stokes (1998) have performed a group-theoretical analysis of simple tilt systems that can be described in terms of basic tilts around the pseudo-cubic axes. Eight of Glazer's tilt systems were found to be redundant due to the fact they impose a higher symmetry than is required by the space-group symmetry. Using this approach, there are 15 tilt systems that can occur in real crystals, each with a different space group. The 15 tilt systems with the space group, degrees of freedom, number of Wyckoff sites for each ion and the estimated number of experimentally reported structures (obtained via a comprehensive literature search) are shown in Table 1. Recently Aleksandrov \& Bartolome (2001) published a very comprehensive review of octahedral tilting distortions in perovskites as well as perovskite-related structures.

\section{Calculation methods}

The software program SPUDS requires only the composition and oxidation state of each ion as its input. The software program executes the entire optimization procedure in approximately $30 \mathrm{~s}$ on an Intel ${ }^{\mathbb{R}}$ Pentium ${ }^{\mathbb{R}}$ class processor using the Microsoft ${ }^{\mathbb{R}}$ Windows ${ }^{\circledR}$ operating system, for which the downloadable version of the software is compiled. The software program was also compiled and executed on other platforms including several types of Unix-based operating systems. A flowchart describing the operation of SPuDS is shown in Fig. 3 and details are provided in the following paragraphs.

To optimize the structure in an unconstrained manner requires determination of the unit-cell dimensions and all free positional parameters. The exact number of variables that must be optimized varies from 3 to 10, depending upon the tilt system (see Table 1). In order to simplify this process $S P u D S$ restricts the octahedra to remain rigid (six equivalent $B-X$ distances and all $X-B-X$ angles equal to $90^{\circ}$ ). This seems to be a reasonable restriction in light of the fact that most distorted perovskites show very little distortion of the $B X_{6}$ octahedra, although obviously $S P u D S$ will not work well in systems where octahedral distortions are expected. Once this restriction is in place the full crystal structure can be generated from two variables: the size of the octahedron and the magnitude of the octahedral tilting distortion. Additional degrees of freedom must be taken into consideration in those tilt systems where either the $A$ cation does not sit on a fixed position $\left(\right.$ e.g. $\left.a^{-} b^{+} a^{-}\right)$or octahedral distortions cannot be avoided $\left(a^{+} a^{+} c^{-}\right)$.

The space groups, approximate unit-cell size, cation and anion positions of each tilt system have been previously derived (Woodward, 1997c). The lattice parameters are based on the linear distance between $B$ cations and decrease as the tilt angle increases. The equations for determining the $X$ positions and lattice parameters based on tilt angle for tilt systems $a^{+} a^{+} a^{+}, a^{-} a^{-} a^{-}, a^{0} b^{+} b^{+}$and $a^{-} b^{+} a^{-}$are taken as derived by O'Keeffe \& Hyde (1977). The equations for the remaining tilt systems are derived geometrically as a function of the octahedral tilt angle and are listed in Table 2. The symmetry information associated with each space group and the atomic positions determined by the tilt equations are needed in order to determine the bond lengths and generate a complete crystallographic description of the structure. SPUDS optimizes the structure by incrementally changing the tilt angle and evaluating the stability of the resulting structure (as described below) at each step. The initial optimization is coarse to allow a wide range of structures to be calculated quickly and the tilt angle increment is finer for successive (35 ) optimization routines resulting in a determination of the optimal tilt angle.

The size of the octahedron and the optimum magnitude of the octahedral tilting distortion is calculated utilizing the bond-valence model, which is used to quantitatively describe inorganic bonding in ionic solids (Brown, 1978). The bond valence, $s_{i j}$, associated with each cation-anion interaction is calculated using (1), where $d_{i j}$ is the cation-anion distance

$$
s_{i j}=e^{\left[\left(R_{i j}-d_{i j}\right) / B\right]} .
$$

The $B$ parameter is empirically determined, but can often be treated as a universal constant with a value of 0.37 . $R_{i j}$ is empirically determined for each cation-anion pair based upon a large number of well determined bond distances for the cation-anion pair in question. Values of $R_{i j}$ for oxides and fluorides can be found in the literature (Brown \& Altermatt, 1985 ; Brese \& O'Keeffe, 1991). The atomic valences, $V_{i(\text { calc) }}$, of 
the $A$ and $B$ cations, and $X$ anion are calculated according to (2) by summing the individual bond valences $\left(s_{i j}\right)$ about each ion

$$
V_{i(\mathrm{calc})}=\sum_{j} s_{i j} .
$$

Six nearest-neighbor anions are used for the $B$ cation, six nearest-neighbor cations for the $X$ anion and 12 nearestneighbor anions for the $A$-site cation are used in the calculations. No assumption is made about the coordination number of the $A$-site cation (valences for $12 A-X$ interactions are calculated in all cases), but the contribution to the atomic valence sum becomes smaller as the $A-X$ bond distance increases. The $B$ cations remain at fixed positions in all space groups generated by simple tilting of the $B X_{6}$ octahedra. The $B-X$ bond distance, which determines the size of the octahedron, is calculated so as to optimize the bond-valence sum of the octahedral cation. The $X-B-X$ bond angles of the $B X_{6}$ octahedra remain ideal $\left(90^{\circ}\right)$ and the $B-X$ bond distances are held constant in the calculations (with the exception of the tilt system $a^{+} a^{+} c^{-}$, which is discussed in $\$ 5.5$ ). The valence sum of the $A$-site cation is varied by changing the magnitude of the octahedral tilting distortion.

The optimized structure is one where the difference between the calculated bond-valence sum and the formal valence (equal to its oxidation state) of each ion is minimized. This value, which is termed the discrepancy factor $d_{i}$ (Rao et al., 1998), is a measure of the lattice strains present in the compound. The discrepancy factor is calculated according to (3), where $V_{i(\mathrm{ox})}$ is the formal valence and $V_{i(\mathrm{calc})}$ is the calculated bond-valence sum for the $i$ th ion

$$
d_{i}=V_{i(\mathrm{ox})}-V_{i(\mathrm{calc})} \text {. }
$$

The overall structure stability is determined by comparing the calculated bond-valence sums with the ideal formal valences. This quantity is referred to as the global instability index (GII; Salinas-Sanchez et.al., 1992) and is calculated according to (4)

$$
G I I=\left\{\left[\sum_{i=1}^{N}\left(d_{i}^{2}\right)\right] / N\right\}^{1 / 2} .
$$

The variables involved in (4) are the discrepancy factor $\left(d_{i}\right)$ and $N$, which is the number of atoms in the asymmetric unit. During the optimization process the octahedral tilt angle is stepped incrementally and the individual $A-X$ and $B-X$ bond distances, discrepancy factors and global instability index are calculated at each step. After the first optimization process, the procedure is repeated using smaller tilt angle increments in order to minimize the GII. The stability of perovskite compositions with different atoms, symmetry, tilt systems and structure can be evaluated by comparing the GII. The GII value is typically $<0.1$ v.u. (valence units) for unstrained structures and as large as 0.2 v.u. in a structure with lattice-induced strains. Crystal structures with a GII greater than 0.2 v.u. are typically found to be unstable and reports of such structures are usually found to be incorrect (Rao et al., 1998).
In certain tilt systems (e.g. $a^{-} b^{+} a^{-}$and $\left.a^{+} a^{+} c^{-}\right)$the $A$-cation position has one or more free positional parameters, so that the $A-X$ distances are not uniquely determined by the tilt angle. This introduces additional degrees of freedom to the optimization process. In these tilt systems the position of the $A$-site cation was optimized according to the following procedure:

(i) The octahedral tilt angle is adjusted in order to minimize the $G I I$ with the $A$-site ions located at their highest symmetry positions (in the center of the cube defined by the eight surrounding octahedral cations).

(ii) Each $A-X$ bond valence is treated as a vector quantity. The magnitude of each valence vector is set equal to the valence of that particular bond and the direction of the valence vector is set parallel to the bond.

(iii) The $12 A-X$ valence vectors are summed and the position of the $A$-site cation is adjusted in order to minimize the magnitude of the resultant vector.

(iv) The octahedral tilt angle is adjusted again in order to minimize the $G I I$ for the new $A$-site cation position.

(v) Steps (ii)-(iv) are repeated until both the $G I I$ and the $A$ site valence vector sum are minimized.

This optimization approach weights the shorter bonds more heavily in determining the $A$-site cation position. This has the effect of moving the $A$-site cation to the most symmetrical coordination environment available within the distorted anion framework. This is exactly the environment that would be expected for $A$-site cations that typically occupy a spherical coordination environment with a small deviation in the shortest to longest $A-X$ bond length. A contour plot of the calcium bond-valence sum over a range of fractional positions in the orthorhombic Pnma structure of $\mathrm{CaTiO}_{3}$ is shown in Fig.

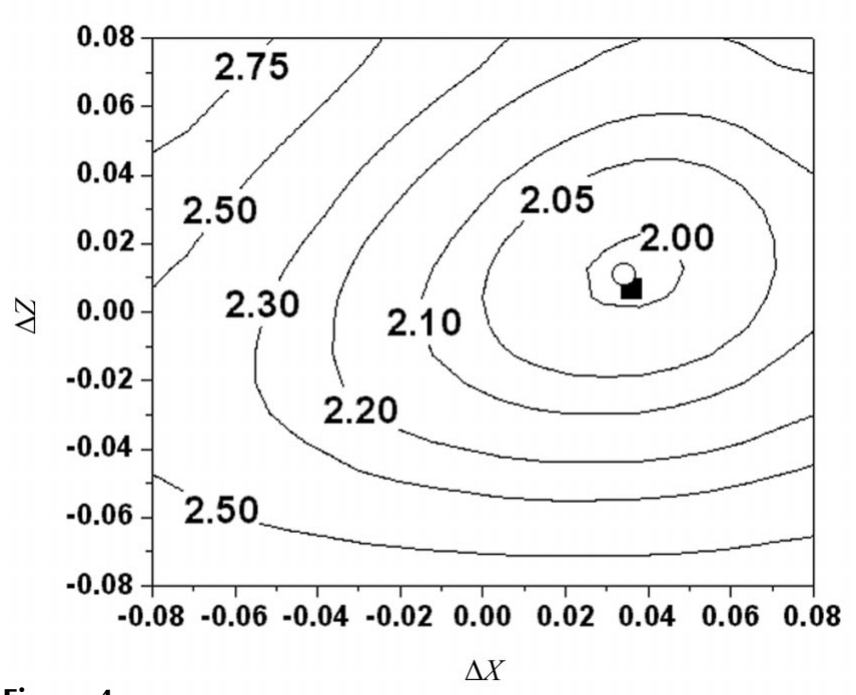

Figure 4

Valence map contour plot of the $A$-site cation for $\mathrm{CaTiO}_{3}$ in tilt system $a^{-} b^{+} a^{-}$(space group Pnma). $\Delta X$ and $\Delta Z$ are the differences in the fractional position from the high-symmetry position located at $\left(\frac{1}{2}, \frac{1}{4}, \frac{1}{2}\right)$. The valence of the $A$-site cation is shown as the free positional parameters are varied while holding the octahedral tilt angle at $14.60^{\circ}$. The open circle is the $S P U D S$ predicted position and the filled square is the literature position. 
4. This clearly shows the accuracy of this approach to positioning the $A$-site cation.

The distribution of tilt systems found in the literature is shown in Fig. 1. The structures were mainly obtained from the earlier work of Woodward (1997b), but additions have been made and are listed in Table 3. The distribution shown in Fig. 1 is naturally biased by trends in scientific research (e.g. superconductivity and magnetoresistance), as well as the fact that the vast majority of structure determinations are carried out at room temperature. However, we feel that Fig. 1 accurately depicts the reported distribution of octahedral tilting distortions in perovskites at room temperature (excluding perovskites which contain multiple cations on the octahedral site). Distorted perovskites can be divided into three groups listed in Table 1. Group A are high-symmetry tilt systems where all $A$-cation sites are crystallographically equivalent, group $\mathrm{B}$ are tilt systems with multiple crystallographic sites for the $A$ cations and group $\mathrm{C}$ are low-symmetry/transitional tilt systems that are often observed as intermediates in a phase transition between two of the higher symmetry structures. The octahedral tilting in groups $\mathrm{A}$ and $\mathrm{B}$ can be described using the notation of Zhao et al. (1993) by a single tilt (e.g. tilting in $a^{0} a^{0} c^{-}$corresponds to a single tilt $\varphi$ about the cubic [001] direction, $a^{0} b^{-} b^{-}$to a single tilt $\theta$ about the cubic [110] and $a^{-} a^{-} a^{-}$to a tilt $\Phi$ about the cubic [111]) or two tilts (e.g. $\left.a^{+} a^{+} c^{-}\right)$at most. The majority of the perovskite structures belong to either group A or B, while structures that fall into group $\mathrm{C}$ are very uncommon. Therefore, the current version of SPUDS calculates structures only for the six high-symmetry and four multiple $A$-site tilt systems and does not calculate structural information for the five low-symmetry/transitional tilt systems. The output file generated by $S P u D S$ contains information including the space group, lattice parameters, atomic coordinates, atomic valence sums, individual bond valences and distances, tolerance factor, unit-cell volume, octahedral tilt angles, $B-X-B$ bond angle and $G I I$ for each of the evaluated tilt systems.

\section{Tolerance factor}

The Goldschmidt tolerance factor (Goldschmidt, 1926) is a measure of the fit of the $A$-site cation to the cubic cornersharing octahedral network. In a cubic perovskite twice the $B-X$ bond length is the cell edge and twice the $A-X$ bond length is equal to the face diagonal. The tolerance factor is shown in (5)

$$
t=\left(R_{A}+R_{X}\right) /\left[2^{1 / 2}\left(R_{B}+R_{X}\right)\right] .
$$

The variable $\tau$ is the tolerance factor, $R_{A}, R_{B}$ and $R_{X}$ are the ionic radii of the $A$ cation, $B$ cation and $X$ anion, respectively. This geometrical relationship is unity for a perovskite structure with an $A$-site cation if the lattice is treated as an array of close-packed spheres. Shannon (1976) has tabulated ionic radii for a variety of coordination environments and oxidation states. The tolerance factor equation requires the use of 12coordinate radii, but unfortunately 12 -coordinate radii are not available for all $A$-site cations. Therefore, extrapolation is
Table 3

Compounds in the $a^{-} b^{+} a^{-}$(Pnma) tilt system not included in the reference by Woodward $(1997 b)$.

\begin{tabular}{ll}
\hline Compound & Reference \\
\hline $\mathrm{CaIrO}_{3}$ & Sarkozy et al. $(1974)$ \\
$A \mathrm{OsO}_{3}(A=\mathrm{Ca}, \mathrm{Sr})$ & Shaplygin \& Lazarev $(1976)$ \\
$A \mathrm{AlO}_{3}(A=\mathrm{Lu}, \mathrm{Yb})$ & Anan'eva et al. $(1978)$ \\
$A \mathrm{VO}_{3}(A=\mathrm{Dy}, \mathrm{Gd})$ & Pickardt et al. $(1988)$ \\
$A \mathrm{RuO}_{3}(A=\mathrm{La}, \mathrm{Pr})$ & Kobayashi et al. $(1994)$ \\
$\mathrm{PrGaO}_{3}$ & Marti et al. $(1994)$ \\
$A \mathrm{AlO}_{3}(A=\mathrm{Eu}, \mathrm{Gd}, \mathrm{Er}, \mathrm{Tb})$ & Shishido et al. $(1995)$ \\
$\mathrm{SrPrO}_{3}$ & Hinatsu \& Itoh $(1996)$ \\
$\mathrm{YCoO}_{3}$ & Mehta et al. $(1997)$ \\
$\mathrm{ScCrO}_{3}$ & Park \& Parise $(1997)$ \\
$\mathrm{SrHfO}_{3}$ & Kennedy et al. $(1999)$ \\
$\mathrm{LaGaO}_{3}$ & Howard \& Kennedy $(1999)$ \\
$\mathrm{ScAlO}_{3}$ & Ross (1998) \\
$\mathrm{CaPbO}_{3}$ & Yamamoto et al. $(1999)$ \\
$A \mathrm{NiO}_{3}(A=\mathrm{Dy}, \mathrm{Eu}, \mathrm{Gd})$ & Alonso et al. $(1999)$ \\
$\mathrm{NdGaO}_{3}$ & Vaselechko et al. $(1999)$ \\
$A \mathrm{MnO}_{3}(A=\mathrm{Er}, \mathrm{Dy}, \mathrm{Ho}, \mathrm{Y})$ & Alonso et al. $(2000)$ \\
$\mathrm{LaMO}_{3}(M=\mathrm{Ho}, \mathrm{Er}, \mathrm{Tm}, \mathrm{Yb}, \mathrm{Lu})$ & Ito et al. $(2001)$ \\
${\mathrm{Ce} M \mathrm{O}_{3}}_{3}(M=\mathrm{Tm}, \mathrm{Yb}, \mathrm{Lu})$ & Ito et al. $(2001)$ \\
$\mathrm{Pr} M O_{3}(M=\mathrm{Yb}, \mathrm{Lu})$ & Ito et al. (2001) \\
\hline
\end{tabular}

necessary to obtain 12-coordinate radii for several ions. The ionic radii calculated tolerance factor uses 12-coordinate $A$ site cation radii, six-coordinate $B$ cation and two-coordinate $X$ anion radii.

Alternatively, one can use the bond-valence model to calculate the ideal $A-X$ and $B-X$ bond distances, assuming 12 equidistant $A-X$ bonds and six equidistant $B-X$ bonds. These $A-X$ and $B-X$ bond distances are then substituted in place of the sum of the ionic radii used in (5) in order to calculate a bond-valence based tolerance factor. SPuDS uses both the ionic radii and the bond-valence parameters separately to calculate the tolerance factor (whenever possible). The use of the bond-valence parameters for the ionic radii does not require any assumption of the coordination environment, only the oxidation state and coordination number are required. A comparison of ionic-radii calculated tolerance factor and bond-valence calculated tolerance factor is shown in Fig. 5. The bond-valence tolerance factor is generally

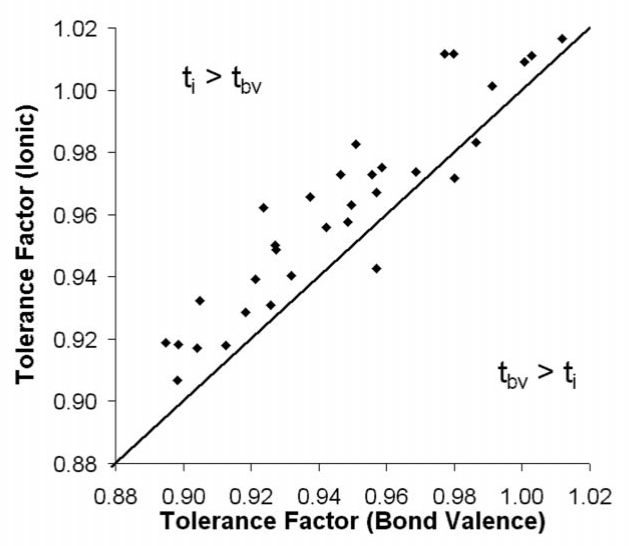

Figure 5

Bond valence and ionic radii calculated tolerance factor with the solid line representing $t_{i}=t_{\mathrm{bv}}$, where $i=$ ionic and $\mathrm{bv}=$ bond valence. 
smaller than the ionic-radii calculated tolerance factor. The ionic-radii calculated tolerance factor is included in $S P u D S$ for comparison to previous investigations. However, all further references to tolerance factors in this paper will correspond to the bond-valence tolerance factor, unless noted otherwise.

\section{Evaluation of known structures using SPuDS}

\section{1. $a^{-} b^{+} a^{-}$(Pnma)}

As shown in Fig. 1 the most common space group for simple perovskites is Pnma, corresponding to the $a^{-} b^{+} a^{-}$tilt system. This structure is also commonly referred to as the $\mathrm{GdFeO}_{3}$ structure. The true crystallographic cell has $Z=4$, with $a \simeq c \simeq$ $2^{1 / 2} a_{p}$ and $b \simeq 2 a_{p}$ (where $a_{p}$ is the unit cell edge length for the undistorted cubic perovskite). The $a^{-} b^{+} a^{-}$tilt system maximizes the $A-X$ covalent bonding and minimizes the repulsive $A-X$ overlap (Woodward, 1997b). This distortion is most common when the (ionic radii) tolerance factor becomes less than 0.98 or when the $A$ site cation becomes relatively elec-

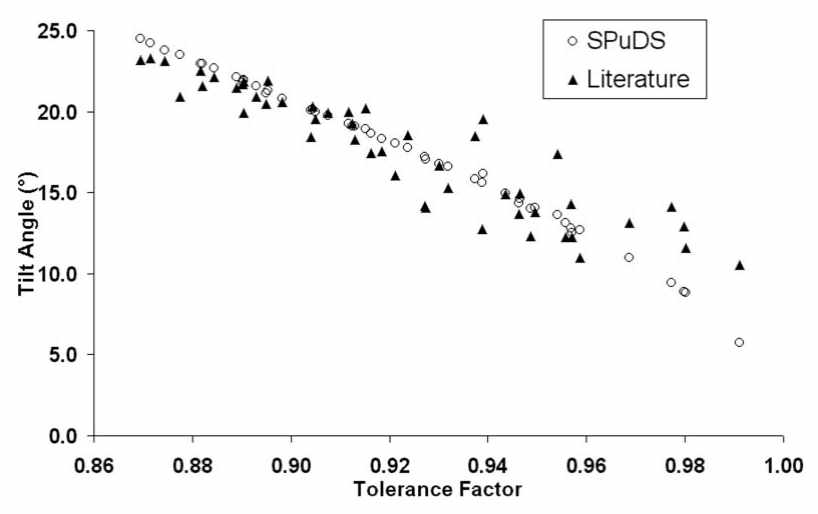

Figure 6

Octahedral tilt angle obtained from the optimized SPuDS structure versus the bond-valence tolerance factor for tilt system $a^{-} b^{+} a^{-}$.

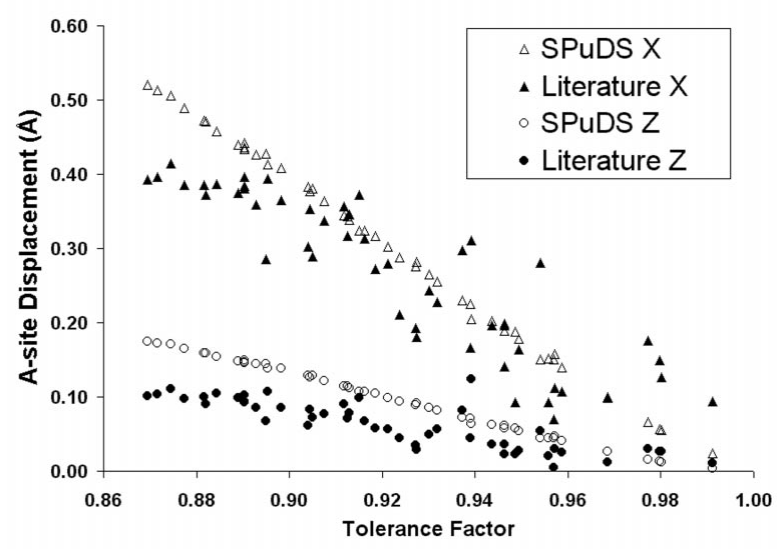

Figure 7

$A$-site cation displacement from high-symmetry location in $x$ and $z$ obtained from the optimized SPuDS structure versus the bond-valence tolerance factor for the tilt system $a^{-} b^{+} a^{-}$. tronegative $\left(\right.$ e.g. $\left.\mathrm{Ca}^{2+}\right)$. The symmetry of the Pnma space group is such that there are ten variables (five fractional coordinates corresponding to the oxygen positions, two for the $A$-site cation, and three variables defining the size of the orthorhombic unit cell), which must be specified in order to completely describe the crystal structure.

In order to assess the accuracy of SPuDS, structures were predicted for a collection of structurally characterized Pnma perovskites. The structural information was tabulated and the experimental and calculated octahedral tilt angles are plotted

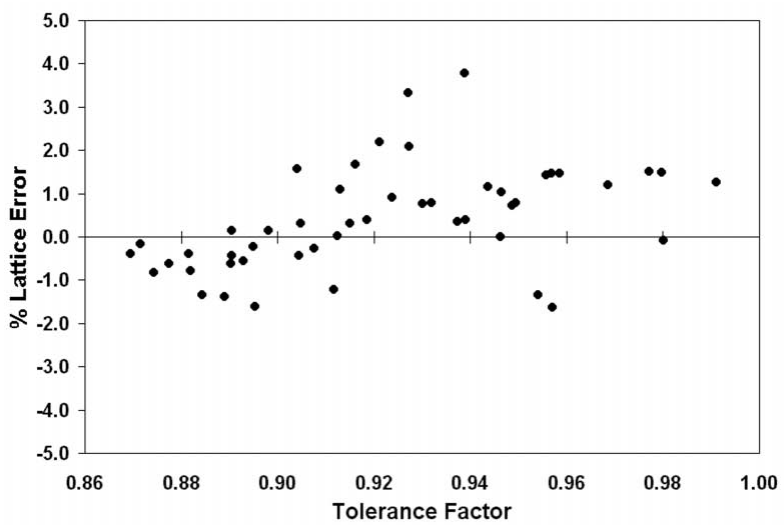

(a)

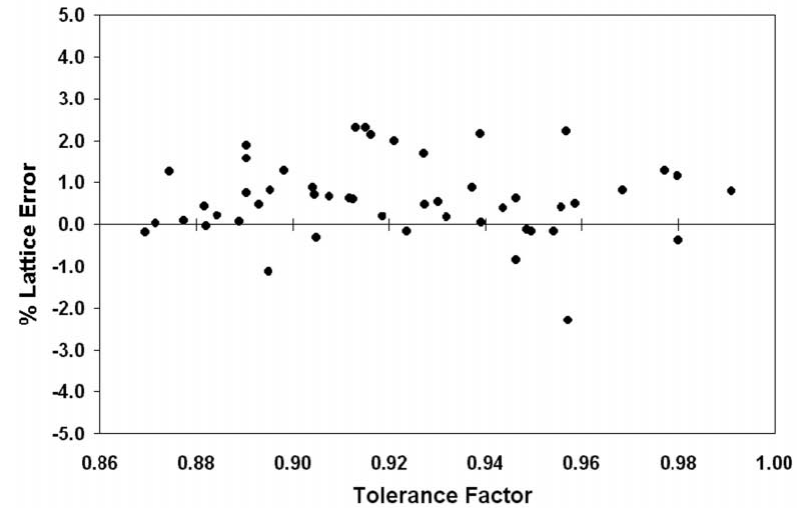

(b)

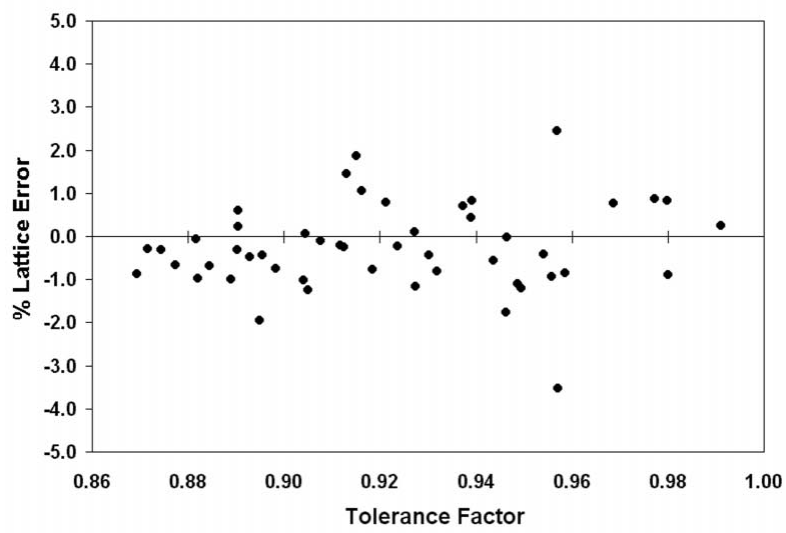

(c)

Figure 8

Percent lattice parameter error $[100 \times(S P u D S$ predicted - experimental)/experimental] versus the bond-calculated tolerance factor for tilt system $a^{-} b^{+} a^{-}$. 
Table 4

Experimental and predicted structural information for typical perovskites crystallizing in the Pnma space group.

\begin{tabular}{|c|c|c|c|c|c|c|c|c|c|c|c|}
\hline \multirow[b]{2}{*}{ Formula } & \multirow[b]{2}{*}{$A 4(c) x$} & \multirow[b]{2}{*}{$A 4(c) z$} & \multirow[b]{2}{*}{ O $4(c) x$} & \multirow[b]{2}{*}{$\mathrm{O} 4(c) z$} & \multirow[b]{2}{*}{$\mathrm{O} 8(d) x$} & \multirow[b]{2}{*}{$\mathrm{O} 8(d) y$} & \multirow[b]{2}{*}{$\mathrm{O} 8(d) z$} & \multirow[b]{2}{*}{ Magnitude tilt angle $\left({ }^{\circ}\right)$} & \multicolumn{3}{|c|}{ Lattice parameters $(\AA)$} \\
\hline & & & & & & & & & $a$ & $b$ & $c$ \\
\hline \multicolumn{12}{|c|}{ Literature atomic fractional coordinates } \\
\hline $\mathrm{GdFeO}_{3}$ & 0.563 & 0.516 & -0.033 & 0.400 & 0.302 & 0.051 & 0.696 & 20.30 & 5.611 & 7.669 & 5.349 \\
\hline $\mathrm{CaTiO}_{3}$ & 0.536 & 0.507 & -0.016 & 0.429 & 0.289 & 0.037 & 0.711 & 14.96 & 5.442 & 7.640 & 5.380 \\
\hline $\mathrm{SrZrO}_{3}$ & 0.524 & 0.504 & -0.013 & 0.427 & 0.285 & 0.035 & 0.716 & 13.69 & 5.817 & 8.171 & 5.796 \\
\hline $\mathrm{SrSnO}_{3}$ & 0.512 & 0.499 & -0.010 & 0.426 & 0.285 & 0.037 & 0.713 & 14.31 & 5.681 & 7.906 & 5.532 \\
\hline \multicolumn{12}{|c|}{$S P u D S$ atomic fractional coordinates } \\
\hline $\mathrm{GdFeO}_{3}$ & 0.568 & 0.524 & -0.021 & 0.394 & 0.299 & 0.053 & 0.697 & 20.13 & 5.587 & 7.723 & 5.352 \\
\hline $\mathrm{CaTiO}_{3}$ & 0.534 & 0.511 & -0.016 & 0.425 & 0.286 & 0.038 & 0.712 & 14.60 & 5.499 & 7.688 & 5.379 \\
\hline $\mathrm{SrZrO}_{3}$ & 0.534 & 0.511 & -0.011 & 0.426 & 0.285 & 0.037 & 0.713 & 14.36 & 5.817 & 8.136 & 5.694 \\
\hline $\mathrm{SrSnO}_{3}$ & 0.526 & 0.508 & -0.008 & 0.434 & 0.282 & 0.033 & 0.717 & 12.80 & 5.765 & 8.082 & 5.668 \\
\hline
\end{tabular}

versus the bond-valence tolerance factor in Fig. 6. The literature octahedral tilt angle was calculated from the reported fractional coordinates of the anion sites. Except for compositions where the tolerance factor approaches unity, $S P u D S$ is able to predict the tilt angle with a high degree of accuracy.

The $A$-site cation positions calculated by $S P u D S$ are compared to literature positions in a graph of the $A$-site cation displacement (in terms of absolute displacement from the center of the simple cubic unit cell) versus tolerance factor in Fig. 7. The $A$-site cation shift in Pnma perovskites is driven by the tilting of the octahedra. The equilibrium position of the $A$ site cation within the tilted octahedral framework represents the most symmetric coordination environment that can be attained for the $A$-site cation. The valence vector mapping approach attempts to replicate this placement of the $A$-site cation. First of all we note that $S P U D S$ displaces the $A$-site cation off the undistorted location in the same direction as observed experimentally. In general the prediction is accurate, but at tolerance factors near unity $S P U D S$ tends to underestimate the magnitude of the $A$-site cation movement, while for large tilt angles the shift of the $A$-site cation is slightly overestimated. The lattice parameters are dependent upon the magnitude of the tilting distortion and the $B-X$ bond distances. The accuracy of lattice parameter prediction is demonstrated in a plot of percent error in lattice parameter versus tolerance factor (see Fig. 8). The average percent error in the lattice parameter for perovskites in the $a^{-} b^{+} a^{-}$tilt system is $1.0 \%$ for $a, 0.9 \%$ for $b$ and $0.8 \%$ for $c$ lattice parameters.

To give the reader a better feel for the absolute accuracy of our approach, predicted structures were examined in detail for four specific perovskites $\mathrm{GdFeO}_{3}$ (Marezio et al., 1970), $\mathrm{CaTiO}_{3}$ (Sasaki et al., 1987), $\mathrm{SrZrO}_{3}$ (Kennedy et al., 1999a) and $\mathrm{SrSnO}_{3}$ (Vegas et al., 1986). Each of these perovskites crystallizes in the Pnma space group, and the experimental and predicted structural information is shown for comparison in Table 4. The experimentally determined structures in the literature are often solved using the nonstandard space group Pbnm or Pcmn. The atomic positions from the original experimental structures were converted to the space group Pnma and to an equivalent Wyckoff atomic position as calculated in SPUDS to allow easier comparison between

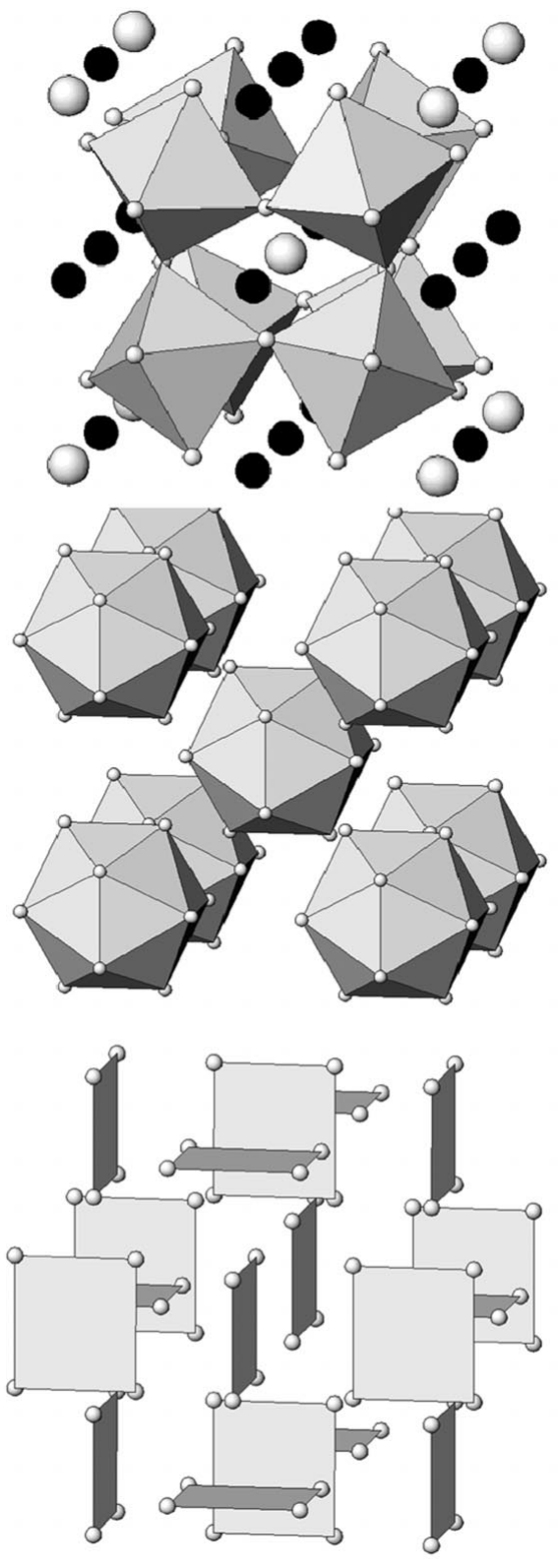

Figure 9

The crystal structure of $\mathrm{CaCu}_{3} \mathrm{Ti}_{4} \mathrm{O}_{12}$, showing the octahedral environment of $\mathrm{Ti}^{4+}$ (top), the icosahedral environment of $\mathrm{Ca}^{2+}$ (middle) and the square planar environment of $\mathrm{Cu}^{2+}$ (bottom). 
Table 5

Bond-valence sums, global instability index, lattice parameter, oxygen free positional parameters, octahedral tilt angle and synthesis pressure for known $\mathrm{CaCu}_{3} M_{4} \mathrm{O}_{12}(M=\mathrm{Ge}, \mathrm{Mn}, \mathrm{Ru}, \mathrm{Ti})$ perovskites reported in the $a^{+} a^{+} a^{+}$tilt system.

Values in italics represent experimentally observed values.

\begin{tabular}{|c|c|c|c|c|c|c|c|c|c|c|}
\hline \multirow[b]{2}{*}{$M$} & \multicolumn{4}{|c|}{ Bond-valence sums (v.u.) } & \multirow[b]{2}{*}{$G I I$ (v.u.) } & \multirow[b]{2}{*}{$a(\AA)$} & \multirow[b]{2}{*}{$\mathrm{O}(y)$} & \multirow[b]{2}{*}{$\mathrm{O}(z)$} & \multirow[b]{2}{*}{ Tilt angle $\left({ }^{\circ}\right)$} & \multirow[b]{2}{*}{ Synthesis pressure (kbar) } \\
\hline & $\mathrm{Ca}$ & $\mathrm{Cu}$ & $M$ & $\mathrm{O}$ & & & & & & \\
\hline \multirow[t]{2}{*}{$\mathrm{Ge}$} & 2.35 & 1.93 & 4.00 & 2.01 & 0.084 & 7.265 & 0.2977 & 0.1910 & 20.7 & $50-70$ \\
\hline & & & & & & 7.202 & 0.3012 & 0.1859 & 20.7 & \\
\hline \multirow[t]{2}{*}{$\mathrm{Mn}$} & 2.33 & 1.94 & 4.00 & 2.01 & 0.078 & 7.283 & 0.2979 & 0.1908 & 20.8 & 50 \\
\hline & & & & & & 7.241 & 0.3033 & 0.1822 & 20.9 & \\
\hline \multirow[t]{2}{*}{$\mathrm{Ru}$} & 1.93 & 2.02 & 4.00 & 2.00 & 0.019 & 7.472 & 0.3050 & 0.1795 & 24.2 & 2 \\
\hline & & & & & & 7.421 & - & - & - & \\
\hline \multirow[t]{2}{*}{$\mathrm{Ti}$} & 2.01 & 2.00 & 4.00 & 2.00 & 0.003 & 7.426 & 0.3036 & 0.1818 & 23.5 & Ambient \\
\hline & & & & & & 7.391 & 0.3038 & 0.1786 & 23.5 & \\
\hline
\end{tabular}

experimental and SPUDS predicted structures. The calculations performed using SPuDS illustrate the accuracy of this approach in predicting the structural trends in the $a^{-} b^{+} a^{-}$tilt system. When examined in this fashion one can see that in an absolute sense the fractional coordinates are determined more accurately than the lattice parameters. This can be attributed to the well known fact that lattice parameters are very sensitive to distortions of the octahedra.

\section{2. $a^{+} a^{+} a^{+}(I m \overline{3})$}

Perovksites which undergo an $a^{+} a^{+} a^{+}$octahedral tilting distortion crystallize in the cubic space group $\operatorname{Im} \overline{3}$. The octahedra tilt in-phase an equivalent amount about each of the three cubic axes. The general formula for structures found in this tilt system is $A^{\prime} A^{\prime \prime}{ }_{3} B_{4} \mathrm{O}_{12}$. The $A$-site cations are at fixed positions in this tilt system, with $A^{\prime}$ and $A^{\prime \prime}$ having two different coordination environments. The $A^{\prime}$ cation is at an icosahedral site with 12 equidistant anions and the coordination environment remains symmetric and contracts rather slowly as the octahedra tilt. The $A^{\prime \prime}$ cation is at a square planar site that contracts much more rapidly as the octahedral tilt angle increases. A Jahn-Teller ion is well adapted for this coordination environment and all known examples of $a^{+} a^{+} a^{+}$ perovskites contain either $\mathrm{Cu}^{2+}$ or $\mathrm{Mn}^{3+}$ on the $A^{\prime \prime}$ site. The coordination environments of both the $A$-site cations are shown in Fig. 9.

Perovskites in this tilt system are often synthesized under high pressure. Perovskites of the formula $\mathrm{CaCu}_{3} M_{4} \mathrm{O}_{12}(M=$ $\mathrm{Ge}, \mathrm{Mn}, \mathrm{Ti}, \mathrm{Ru}$ ) have been synthesized (Ozaki et al., 1977; Bochu et al., 1979; Deschanvres et al., 1967; Labeau et al., 1980). A sodium containing perovskite $\mathrm{NaMn}_{7} \mathrm{O}_{12}$ (Marezio et $a l ., 1973)$ has also been made. Recently it has been shown that the perovskite $\mathrm{CaCu}_{3} \mathrm{Ti}_{4} \mathrm{O}_{12}$ exhibits fascinating behavior as a dielectric material. A rather high dielectric constant of approximately 12000 at $1 \mathrm{kHz}$ has been recently observed and is nearly constant from room temperature to $573 \mathrm{~K}$ (Subramanian et al., 2000). The dielectric constant lowers nearly 100fold near $100 \mathrm{~K}$, with no apparent structural transition. The origin of this effect and its mechanism are still not well understood (Ramirez et al., 2000).

The $\mathrm{CaCu}_{3} M_{4} \mathrm{O}_{12}(M=\mathrm{Ge}, \mathrm{Mn}, \mathrm{Ti}, \mathrm{Ru})$ formulae were evaluated with $S P U D S$ and the bond-valence sums, GII, lattice parameters, oxygen positions, octahedral tilt angle and synthesis conditions are given in Table 5. The optimal GII in calculated tilt systems are illustrated in Fig. 10. Comparing across all of the calculated tilt systems it is observed that the lowest GII is observed in the $a^{+} a^{+} a^{+}$tilt system. From this data one can see that the global instability index for $\mathrm{CaCu}_{3} \mathrm{Ti}_{4} \mathrm{O}_{12}$ is substantially lower than for the $M=\mathrm{Ge}, \mathrm{Mn}, \mathrm{Ru}$ structures. This observation helps to rationalize the fact that $\mathrm{CaCu}_{3} \mathrm{Ti}_{4} \mathrm{O}_{12}$ is the only compound of the four that can be synthesized at atmospheric pressure. These results also provide some insight regarding the values of GII, which might be expected to result in successful high-pressure synthetic attempts.

\section{3. $\mathbf{a}^{-} \mathbf{a}^{-} \mathbf{a}^{-}(R \overline{3} \mathrm{c})$}

The $a_{-}^{-} a^{-} a^{-}$tilt system crystallizes in the trigonal space group $R \overline{3} c$ with three degrees of freedom. This space group has a single crystallographic site for each of the $A, B$ and $X$ ions. Known compositions in the $a^{-} a^{-} a^{-}$tilt system with exclusively lanthanum as the $A$-site cation are $\mathrm{LaNiO}_{3}$ (García-Muñoz et al., 1992), $\mathrm{LaCuO}_{3}$ (Demazeau et al., 1972), $\mathrm{LaAlO}_{3}$ (Howard, Kennedy \& Chakoumakos, 2000), $\mathrm{LaCoO}_{3}$ (Thornton et al., 1986) and $\mathrm{LaGaO}_{3}$ (Howard \& Kennedy, 1999). The ionic radii tolerance factor is greater than unity (1.003, 1.014, 1.017, 1.011 and 0.973 , respectively) for four out

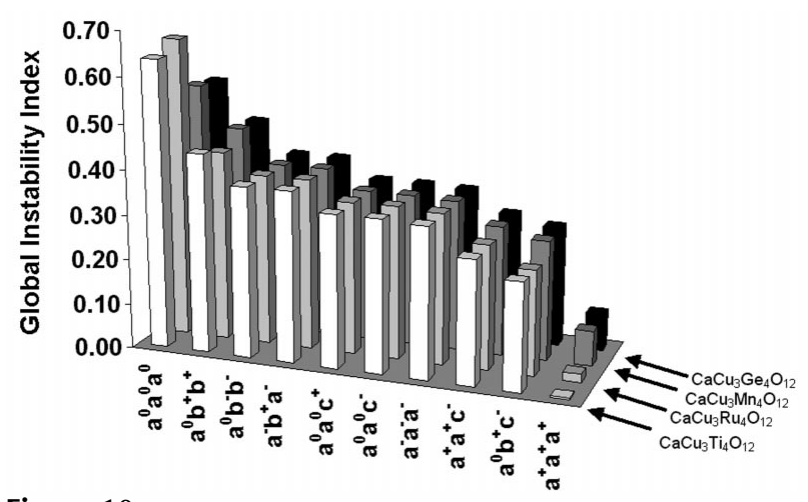

Figure 10

$S P u D S$ modelling for known compounds of the tilt system $a^{+} a^{+} a^{+}$. The calculated GII for the tilt system $a^{+} a^{+} a^{+}$are given in Table 5. The calculated GII for the next lowest tilt system $\left(a^{0} b^{-} c^{+}\right)$are $(0.237$, $\left.\mathrm{CaCu}_{3} \mathrm{Ti}_{4} \mathrm{O}_{12}\right),\left(0.232, \mathrm{CaCu}_{3} \mathrm{Ru}_{4} \mathrm{O}_{12}\right),\left(0.266, \mathrm{CaCu}_{3} \mathrm{Mn}_{4} \mathrm{O}_{12}\right), \quad(0.268$, $\mathrm{CaCu}_{3} \mathrm{Ge}_{4} \mathrm{O}_{12}$ ). 
Table 6

$S P U D S$ predicted and experimental $G I I$, lattice parameters, unit-cell volume, bond-valence sums, bond distances and $\mathrm{O}-\mathrm{Ti}-\mathrm{O}$ bond angles for $\mathrm{CaFeTi}_{2} \mathrm{O}_{6}$.

\begin{tabular}{llllll}
\hline & $S P u D S$ & Literature & Bond distances $(\AA)$ & SPuDS & Literature \\
\hline$G I I($ v.u. $)$ & 0.108 & 0.129 & $\mathrm{Ti}[8(c)]-\mathrm{O}[8(f)](\times 2)$ & 1.97 & 1.97 \\
$a(\AA)$ & 7.59 & 7.52 & $\mathrm{Ti}[8(c)]-\mathrm{O}[8(g)](\times 2)$ & 1.97 & 1.94 \\
$c(\AA)$ & 7.48 & 7.55 & $\mathrm{Ti}[8(c)]-\mathrm{O}[8(g)](\times 2)$ & 1.98 & 1.97 \\
$\mathrm{Unit-cell} \mathrm{volume}\left(\AA^{3}\right)$ & 430.9 & 426.7 & & & 2.10 \\
$\mathrm{Bond-valence} \mathrm{sums} \mathrm{(v.u.)}$ & & & $\mathrm{Fe}[2(a)]-\mathrm{O}[8(f)](\times 4)$ & 2.07 & 3.15 \\
$\mathrm{Fe}[2(a)]$ & 1.90 & 1.79 & $\mathrm{Fe}[2(a)]-\mathrm{O}[8(g)](\times 4)$ & 3.18 & 2.84 \\
$\mathrm{Fe}[2(b)]$ & 1.78 & 1.85 & $\mathrm{Fe}[2(a)]-\mathrm{O}[8(g)](\times 4)$ & 2.86 & 3.22 \\
$\mathrm{Ca}[4(d)]$ & 2.26 & 2.30 & $\mathrm{Fe}[2(b)]-\mathrm{O}[8(f)](\times 4)$ & 3.30 & 2.80 \\
$\mathrm{Ti}[8(c)]$ & 3.91 & 4.05 & $\mathrm{Fe}[2(b)]-\mathrm{O}[8(g)](\times 4)$ & 2.73 & 2.08 \\
$\mathrm{O}[8(f)]$ & 1.97 & 1.97 & $\mathrm{Fe}[2(b)]-\mathrm{O}[8(g)](\times 4)$ & 2.11 & \\
$\mathrm{O}[8(g)]$ & 2.03 & 2.14 & & & 2.72 \\
$\mathrm{O}[8(g)]$ & 1.96 & 2.00 & $\mathrm{Ca}[4(d)]-\mathrm{O}[8(f)](\times 4)$ & 2.77 & 2.33 \\
$\mathrm{O}-B-\mathrm{O}$ bond angle $\left({ }^{\circ}\right)$ & & & $\mathrm{Ca}[4(d)]-\mathrm{O}[8(f)](\times 2)$ & 2.34 & 2.46 \\
$\mathrm{O}[8(f)]-B-\mathrm{O}[8(g)]$ & 90.0 & 89.1 & $\mathrm{Ca}[4(d)]-\mathrm{O}[8(g)](\times 2)$ & 2.48 & 2.51 \\
$\mathrm{O}[8(f)]-B-\mathrm{O}\left[8\left(g^{\prime}\right)\right]$ & 89.9 & 89.0 & $\mathrm{Ca}[4(d)]-\mathrm{O}[8(g)](\times 2)$ & 2.46 & 3.39 \\
$\mathrm{O}[8(g)]-B-\mathrm{O}\left[8\left(g^{\prime}\right)\right]$ & 89.8 & 89.8 & $\mathrm{Ca}[4(d)]-\mathrm{O}[8(g)](\times 2)$ & 3.42 & \\
\hline
\end{tabular}

rigorously evaluating $S P U D S$ predictive capabilities in these tetragonal systems. $\mathrm{CsAgF}_{3}$ and $\mathrm{RbAgF}_{3}$ were evaluated using $S P u D S$, however, the $\mathrm{AgF}_{6}$ octahedra exhibit a significant distortion in $\mathrm{Ag}-\mathrm{F}$ bond lengths and $S P u D S$ is not currently designed to handle this type of distortion. In fact, it may well be that the tetragonal distortion of the octahedra stabilizes the tilting distortion, rather than the other way around.

An additional phase transition was recently determined in $\mathrm{SrZrO}_{3}$ (Howard, Knight et al., 2000), thus, there are six known representatives of the $a^{0} b^{-} b^{-}$tilt system (Imma). The $A$ site cation is $\mathrm{Ba}$ in four of the six structures, thus a large polarizable $A$ -

of the five compounds, so that by simple arguments one might expect an octahedral tilting distortion only for $\mathrm{LaGaO}_{3}$. In contrast, the bond-valence tolerance factor is less than unity (0.994, 0.981, 1.012, 0.970 and 0.956 , respectively) for four of the five compounds. Thus, evaluation using bond valences rather than ionic radii helps to explain why these compounds undergo octahedral tilting distortions in the first place (except for $\mathrm{LaAlO}_{3}$ ). Calculations were carried out on these compounds and compared with experimentally determined structures. The octahedral tilt angle predicted by $S P u D S$ follows a smooth curve over a range of tolerance factors, as one would expect. However, when the experimentally determined crystal structures are examined, a clear relationship between tilt angle and tolerance factor does not appear. The octahedral tilt angle in these compounds appears independent of the geometry-based tolerance factor. This result would seem to indicate that in the $a^{-} a^{-} a^{-}$tilt system something other than the valence requirements of the $A$-site cation drives the octahedral tilting distortion (such as distortions of the octahedra).

\section{4. $a^{0} a^{0} c^{-}(I 4 / m c m), a^{0} a^{0} c^{+}(P 4 / m b m)$ and $a^{0} b^{-} b^{-}$(Imma)}

Most of the compounds that adopt the $a^{0} a^{0} c^{-}$and $a^{0} a^{0} c^{+}$tilt system do so at non-ambient temperatures. $\mathrm{CsAgF}_{3}$ and $\mathrm{RbAgF}_{3}$ (Odenthal \& Hoppe, 1971) structure determinations were performed at room temperature in $14 / \mathrm{mcm}$, but no perovskite oxides are reported in the literature at ambient temperature. Only $\mathrm{CsDyBr}_{3}$ was found in $\mathrm{P} 4 / \mathrm{mbm}$ at room temperature (Hohnstedt \& Meyer, 1993). The calculation methods employed in SPUDS cannot determine which of these two tilt systems are more favorable, since all nearest-neighbor distances are equivalent. The current version of $S P U D S$ does not have temperature-dependent bond-valence parameters and for this reason the predicative ability will be less accurate at temperatures above and below room temperature. The lack of any examples of ambient-condition perovskite oxides that adopt the $a^{0} a^{0} c^{-}$and $a^{0} a^{0} c^{+}$tilt system prevents us from site cation together with a tolerance factor intermediate between Pnma and $P m \overline{3} m$ appear to stabilize the $a^{0} b^{-} b^{-}$tilt system. Simulations of a variety of compounds were performed, but the $a^{-} b^{+} a^{-}$and $a^{-} a^{-} a^{-}$invariably had a lower calculated GII. The $a^{-} b^{+} a^{-}$tilt system has a lower GII due to the additional degrees of freedom and $a^{-} a^{-} a^{-}$has a lower GII due to the symmetric collapse of the octahedra around the $A$ site cation. Clearly additional calculation methods are needed in order to understand the stability of this tilt system in more detail.

\section{5. $a^{+} a^{+} c^{-}\left(P 4_{2} / n m c\right)$}

The symmetry for the Glazer tilt system $a^{+} a^{+} c^{-}$is correctly described in the tetragonal space group $\mathrm{P}_{2} / n m c$ rather than the original assignment of Pmmn (Leinenweber \& Parise, 1995). A vector proof has demonstrated that distortions of the octahedra are necessary to retain corner-sharing connectivity in the tilt system $a^{+} a^{+} c^{-}$(Woodward, 1997c). Howard \& Stokes (1998) subsequently confirmed this conclusion. Thus, our restriction that the octahedra remain rigid cannot be strictly applied in this tilt system. Furthermore, the situation is complicated by the fact that the octahedra can distort in a number of different ways. Our approach to this problem was to determine the positions of the $X 18(f)$ and one of the $X 2$ $8(g)$ atoms directly from the tilt angles (there are two distinct tilt angles). The two free positional parameters of the third $X 3$ anion, also at Wyckoff position $8(g)$, are varied and each of the cis- $X-B-X$ bond angles are calculated. The positional parameters for the third $X$ anion are assigned the values in which the sum of the difference between each $c i s-X-B-X$ bond angle and $90^{\circ}$ is a minimum. Using this approach for determining the position of the third $X$ anion the $B-X\left[8\left(g^{\prime}\right)\right]$ bond length is altered from its ideal value. The calculation method has the effect of allowing a distortion of the octahedral bond lengths, while retaining approximately $90^{\circ} X-B-$ $X$ angles. 
Table 7

Experimental and $S P u D S$ predicted global instability index, lattice parameters (optimized via Bcation valence sum, $A$-cation valence sum and minimized $G I I$ ) for cubic perovskite fluorides and oxides.

\begin{tabular}{|c|c|c|c|c|c|c|c|}
\hline Formula & $\begin{array}{l}\text { Tolerance } \\
\text { factor }\end{array}$ & $\begin{array}{l}\text { Literature } \\
a(\AA)\end{array}$ & $\begin{array}{l}B \text {-cation } \\
\text { valence sum } \\
\text { optimized } \\
a(\AA)\end{array}$ & $\begin{array}{l}A \text {-cation } \\
\text { valence sum } \\
\text { optimized } \\
a(\AA)\end{array}$ & $\begin{array}{l}G I I \\
\text { minimized } \\
a(\AA)\end{array}$ & $\begin{array}{l}G I I \\
\text { (v.u.) }\end{array}$ & Reference \\
\hline $\mathrm{KMgF}_{3}$ & 1.04 & 3.95 & 3.97 & 4.12 & 4.03 & 0.108 & Zhao et al. (1996) \\
\hline $\mathrm{KNiF}_{3}$ & 1.03 & 4.01 & 4.01 & 4.12 & 4.05 & 0.081 & Kijima et al. (1983) \\
\hline $\mathrm{KZnF}_{3}$ & 1.02 & 4.06 & 4.05 & 4.12 & 4.08 & 0.046 & Buttner \& Maslen (1988) \\
\hline $\mathrm{KCoF}_{3}$ & 1.01 & 4.07 & 4.09 & 4.12 & 4.10 & 0.017 & Kijima et al. (1981) \\
\hline $\mathrm{KFeF}_{3}$ & 1.00 & 4.12 & 4.11 & 4.12 & 4.11 & 0.004 & Miyata et al. (1983) \\
\hline $\mathrm{BaLiF}_{3}$ & 1.00 & 4.00 & 4.05 & 4.17 & 4.04 & 0.009 & Zhao et al. (1996) \\
\hline $\mathrm{KMnF}_{3}$ & 0.98 & 4.19 & 4.21 & 4.12 & 4.18 & 0.062 & Kijima et al. (1983) \\
\hline $\mathrm{RbCaF}_{3}$ & 0.97 & 4.45 & 4.50 & 4.35 & 4.45 & 0.095 & Hutton \& Nelmes (1981) \\
\hline $\mathrm{KTaO}_{3}$ & 1.09 & 3.99 & 3.97 & 4.32 & 4.04 & 0.368 & Zhurova et al. (1995) \\
\hline $\mathrm{SrGeO}_{3}$ & 1.04 & 3.80 & 3.80 & 3.93 & 3.86 & 0.199 & Shimizu et al. (1970) \\
\hline $\mathrm{BaNbO}_{3}$ & 1.03 & 4.09 & 4.06 & 4.17 & 4.11 & 0.157 & Svensson \& Werner (1990) \\
\hline $\mathrm{BaMoO}_{3}$ & 1.02 & 4.04 & 4.07 & 4.17 & 4.11 & 0.140 & Brixner (1960) \\
\hline $\mathrm{SrVO}_{3}$ & 1.02 & 3.84 & 3.87 & 3.93 & 3.89 & 0.093 & Rey et al. (1990) \\
\hline $\mathrm{BaSnO}_{3}$ & 1.01 & 4.12 & 4.11 & 4.17 & 4.14 & 0.084 & Smith \& Welch (1960) \\
\hline $\mathrm{KUO}_{3}$ & 1.01 & 4.30 & 4.28 & 4.32 & 4.29 & 0.027 & Dickens \& Powell (1991) \\
\hline $\mathrm{BaZrO}_{3}$ & 1.00 & 4.19 & 4.16 & 4.17 & 4.16 & 0.018 & Roth (1957) \\
\hline $\mathrm{SrTiO}_{3}$ & 1.00 & 3.91 & 3.93 & 3.93 & 3.93 & 0.006 & Hutton et al. (1981) \\
\hline $\mathrm{SrMoO}_{3}$ & 0.97 & 3.97 & 4.07 & 3.93 & 4.03 & 0.186 & Liu et al. (1992) \\
\hline
\end{tabular}

general it will be energetically more favorable to compress the $A-X$ bonds than to stretch the $B-X$ bonds.

\section{Prediction of novel structures}

\section{1. $a^{0} b^{+} b^{+}(14 / \mathrm{mmm})$}

There are no known examples of simple $A B X_{3}$ perovskites that crystallize in the tilt system $a^{0} b^{+} b^{+}$ $(I 4 / \mathrm{mmm})$. The tilt system has three $A$-site cation Wyckoff sites, 2(a), 2(b) and $4(c)$, and five degrees of freedom. The three different $A$-site cation positions enable a large number of $A$ site cation combinations to be evaluated. The $A$-site cation located on the 2(a) Wyckoff position has four short and eight long $A-X$ bonds, the 2(b) Wyckoff position has eight short and four long $A-X$ bonds and the 4(c) Wyckoff position has four short, four medium and four long $A-X$ bonds after octahedral tilting has

Evaluation of SPUDS accuracy for $a^{+} a^{+} c^{-}$perovskites is difficult due to the fact that this tilt system is very uncommon. The only perovskite synthesized in the $a^{+} a^{+} c^{-}$tilt system thus far is $\mathrm{CaFeTi}_{2} \mathrm{O}_{6}$ (Leinenweber \& Parise, 1995). It was synthesized under high-pressure, high-temperature conditions. The unit-cell volumes, global instability indexes, bond distances and valence sums, and $\mathrm{O}-\mathrm{Ti}-\mathrm{O}$ bonds angles for $\mathrm{CaFeTi}_{2} \mathrm{O}_{6}$ are shown in Table 6. The Fe is somewhat underbonded, while the $\mathrm{Ca}$ is over-bonded. The SPuDS calculations show the $a^{+} a^{+} c^{-}$tilt system has the lowest GII of any of the calculated tilt system. The GII of each tilt system for the modelling of $\mathrm{CaFeTi}_{2} \mathrm{O}_{6}$ is shown in Fig. 11.

\section{6. $a^{0} a^{0} a^{0}(P m \overline{3} m)$}

The undistorted cubic perovskite structure has one degree of freedom (the cell edge) to satisfy both the $A-X$ and $M-X$ bonding requirements. In most cases these two bonding interactions will not be perfectly matched and the equilibrium structure will represent a compromise. To evaluate the nature of this compromise we calculated the cell edge of several room-temperature cubic oxide and fluoride perovskites using three different approaches:

(i) optimizing the $B$-cation valence,

(ii) optimizing the $A$-cation valence and

(iii) minimizing the GII.

The results are compared with the observed cell edges in Table 7. The results show that in general the $B-X$ bonding dictates the length of the cell edge. This is particularly true when the $A-X$ interactions are highly ionic and the tolerance factor is much larger than unity. This result is not too surprising considering the fact that highly ionic $A-X$ bonds would be expected to show greater flexibility. Thus, we conclude that in occurred. The 2(a) Wyckoff position has a square planar coordination, so that an atom such as $\mathrm{Cu}^{2+}, \mathrm{Pd}^{2+}$ or $\mathrm{Pt}^{2+}$, which are known to adopt a square planar coordination, will be atoms most likely to occupy this site. A variety of atoms were inserted at the other $A$-site positions in order to obtain a low global instability index. Hypothetical structures were evaluated and the bond-valence sums, GII and octahedral tilt angle of the three most favorable compositions are shown in Table 8 . The global instability index for each of the tilt systems that $S P u D S$ calculates is shown for $\mathrm{PdCdCa}_{2} \mathrm{Ti}_{4} \mathrm{O}_{12}$ in Fig. 11 .

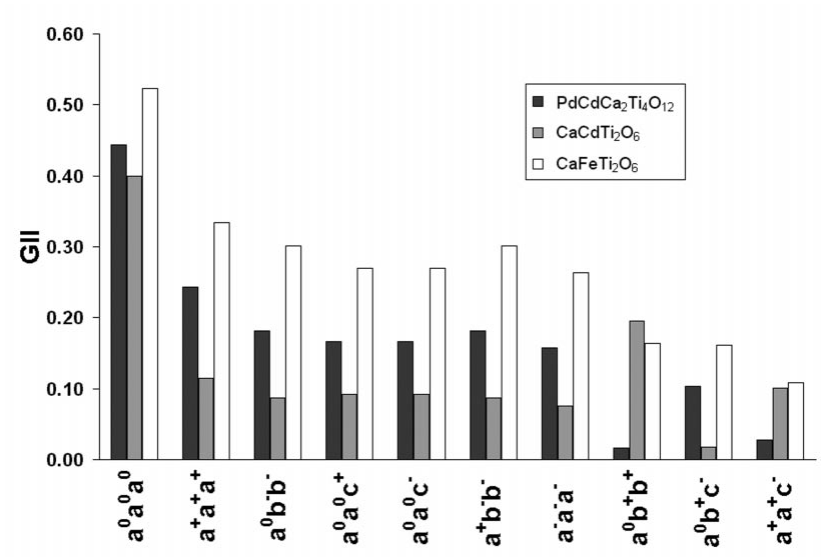

Figure 11

SPUDS predicted GII values for $\mathrm{PdCdCa}_{2} \mathrm{Ti}_{4} \mathrm{O}_{12}, \mathrm{Ca}_{2} \mathrm{Cd}_{2} \mathrm{Ti}_{4} \mathrm{O}_{12}$ and $\mathrm{CaFeTi}_{2} \mathrm{O}_{6}$ in each of the calculated tilt systems. $\mathrm{CaFeTi}_{2} \mathrm{O}_{6}$ was synthesized (Leinenweber \& Parise, 1995) under high-pressure, hightemperature conditions and the structure solved in the $a^{+} a^{+} c^{-}$tilt system. $\mathrm{PdCdCa}_{2} \mathrm{Ti}_{4} \mathrm{O}_{12}$ and $\mathrm{Ca}_{2} \mathrm{Cd}_{2} \mathrm{Ti}_{4} \mathrm{O}_{12}$ are hypothetical compounds designed to adopt tilt systems $a^{0} b^{+} b^{+}$and $a^{0} b^{-} c^{+}$, respectively. The bond-valence sums, GII and tilt angle for $\mathrm{PdCdCa}_{2} M_{4} \mathrm{O}_{12}(M=\mathrm{Ti}, \mathrm{Os}, \mathrm{Ru})$ and $\mathrm{Ca}_{2} \mathrm{Cd}_{2} M_{4} \mathrm{O}_{12}(M=\mathrm{Ti}, \mathrm{Ru}, \mathrm{Ge})$ are given in Tables 8 and 9 , respectively. 
Table 8

Calculated bond-valence sums, $G I I$ and octahedral tilt angle for hypothetical compounds designed to adopt the $a^{0} b^{+} b^{+}$tilt system.

\begin{tabular}{|c|c|c|c|c|c|c|c|c|}
\hline \multirow[b]{2}{*}{ Compound } & \multicolumn{6}{|c|}{ Bond-valence sums (v.u.) } & \multirow[b]{2}{*}{$G I I$ (v.u.) } & \multirow[b]{2}{*}{ Tilt angle $\left(^{\circ}\right)$} \\
\hline & $A[2(a)]$ & $A[2(b)]$ & $A[4(c)]$ & $B$ & $X[8(h)]$ & $X[16(n)]$ & & \\
\hline $\mathrm{PdCdCa}_{2} \mathrm{Ti}_{4} \mathrm{O}_{12}$ & 1.97 & 1.97 & 2.04 & 4.00 & 2.00 & 2.00 & 0.016 & 17.8 \\
\hline $\mathrm{PdCdCa}_{2} \mathrm{Os}_{4} \mathrm{O}_{12}$ & 1.96 & 1.97 & 2.05 & 4.00 & 2.00 & 2.00 & 0.019 & 17.6 \\
\hline $\mathrm{PdCdCa}_{2} \mathrm{Ru}_{4} \mathrm{O}_{12}$ & 2.03 & 1.93 & 2.01 & 4.00 & 2.00 & 2.00 & 0.017 & 18.7 \\
\hline
\end{tabular}

Table 9

Calculated bond valence sums, GII and octahedral tilt angle for hypothetical compounds designed to adopt the $a^{0} b^{-} c^{+}$tilt system.

\begin{tabular}{|c|c|c|c|c|c|c|c|c|}
\hline \multirow[b]{2}{*}{ Compound } & \multicolumn{6}{|c|}{ Bond-valence sums (v.u.) } & \multirow[b]{2}{*}{$G I I$ (v.u.) } & \multirow[b]{2}{*}{ Tilt angle $\left({ }^{\circ}\right)$} \\
\hline & $A 1[4(c)]$ & $A 2[4(c)]$ & $B$ & $X[8(e)]$ & $X[8(f)]$ & $X[8(g)]$ & & \\
\hline $\mathrm{CaCdGe}_{2} \mathrm{O}_{6}$ & 2.12 & 1.90 & 4.00 & 2.01 & 2.01 & 1.99 & 0.052 & 11.5 \\
\hline $\mathrm{CaCdTi}_{2} \mathrm{O}_{6}$ & 2.00 & 1.99 & 4.01 & 2.00 & 2.03 & 1.98 & 0.017 & 16.6 \\
\hline $\mathrm{CaCdRu}_{2} \mathrm{O}_{6}$ & 1.96 & 2.02 & 4.02 & 1.99 & 2.03 & 1.98 & 0.025 & 17.7 \\
\hline
\end{tabular}

\section{Conclusions}

The software program SPUDS has been developed for predicting the structures of perovskite compounds. The optimization procedure is based on the bond-valence method and requires only the composition as user input. Predictions for existing compounds confirm the validity of this approach. $S P U D S$ could be useful for a variety of purposes, such as evaluating the stability and properties of new perovskite materials, and/or generating accurate starting models for structure refinements.

$S P U D S$ is capable of predicting fractional coordinates for members of the $a^{-} b^{+} a^{-}($Pnma $)$and $a^{+} a^{+} a^{+}(\operatorname{Im} \overline{3})$ tilt systems, as well as undistorted

The GII for $a^{0} b^{+} b^{+}$is lower than the other tilt systems, but there is not a large difference between the GII of the next lowest tilt system, $a^{+} a^{+} c^{-}$. The small difference in stability between the two lowest tilt systems implies that there is not a significant driving force to adopt the $a^{0} b^{+} b^{+}$tilt system, although it is worthwhile to note that $a^{+} a^{+} c^{-}$is also a very uncommon tilt system. Furthermore, one must not forget that the formula $\mathrm{PdCdCa}_{2} \mathrm{Ti}_{4} \mathrm{O}_{12}$ in $a^{0} b^{+} b^{+}$is not necessarily the most stable phase for that combination of elements. For example, the stable phase $\mathrm{CaTiO}_{3}$ plus other phases may form instead.

\section{2. $a^{0} b^{-} c^{+}(C m c m)$}

Examples of perovskite oxide structures that have been reported in this space group are all high-temperature polymorphs, including $\mathrm{SrZrO}_{3}$ (970-1100 K; Kennedy et al., 1999a), $\mathrm{NaNbO}_{3}$ (793-848 K; Darlington \& Knight, 1999a,b), $\mathrm{NaTaO}_{3}$ (773-843 K; Kennedy, Prodjosantoso \& Howard, 1999) and $\mathrm{CaTiO}_{3}(1380-1500 \mathrm{~K}$; Kennedy et al., 1999c). There are ten degrees of freedom for this tilt system and two $A$-site cations at Wyckoff position 4(c) with a slightly different coordination. A structure that might crystallize in this tilt system would most likely have two $A$-site cations of similar ionic radius. Using this approach, a wide variety of hypothetical structures were evaluated and examples of the three most promising compositions are shown in Table 9. The calculated global instability indexes were similar for several nearby tilt systems. Hence, there is not a large structural driving force to stabilize this tilt system and multiphase mixtures may well be more stable than a single-phase $a^{0} b^{-} c^{+}$perovskite. The small difference in GII between the different tilt systems provides insight as to why this tilt system is not observed at ambient temperature and only a few compounds are observed in a higher temperature range. perovskites, with a high degree of accuracy. The prediction of unit-cell parameters is not quite as good as the predictions of atomic fractional coordinates owing to the effects of octahedral distortions, but the predicted values are consistently within $1 \%$ of the observed values. For the $a^{+} a^{+} a^{+}$tilt system the GII calculated by SPuDS appears to correlate with the pressure required for phase stabilization and successful synthesis. Clear-cut conclusions cannot be drawn for intermediate tilt systems $\left[a^{0} a^{0} c^{-}, a^{0} a^{0} c^{+}, a^{0} b^{-} b^{-}, a^{-} a^{-} a^{-}\right]$between $a^{-} b^{+} a^{-}$and $a^{0} a^{0} a^{0}$ owing to the relatively small number of representatives in these tilt systems. However, it appears that distortion mechanisms other than octahedral tilting must be taken into account in order to fully understand these systems. The complete absence of compounds that adopt the $a^{0} b^{+} b^{+}$ and $a^{0} b^{-} c^{+}$tilt systems under ambient conditions is a consequence of the fact that the $A$-site coordination environments in these structures are not sufficiently distinct to effectively stabilize $A$-site cation ordering.

Now that the accuracy of this approach has been demonstrated we hope to extend the capabilities of SPuDS to include octahedral tilting in combination with cation ordering $\left(A_{2} B B^{\prime} X_{6}, A_{3} B_{2} B^{\prime} X_{9}, A A^{\prime} B_{2} X_{6}\right)$, Jahn-Teller distortions, anion-vacancy ordering $\left(\operatorname{Ln} A B_{2} \mathrm{O}_{5}, \operatorname{Ln} A_{2} B_{3} \mathrm{O}_{8}, A_{2} B_{2} \mathrm{O}_{5}\right)$ and intergrowth phases (Ruddlesden-Popper, Aurivillius and Dion-Jacobson phases). The software program SPuDS is available by contacting the authors via e-mail (woodward@chemistry.ohio-state.edu or mlufaso@chemistry.ohiostate.edu) or by free download at http://www.chemistry.ohiostate.edu/ mlufaso/spuds/index.html.

The authors would like to thank I. D. Brown for providing the Accumulated Table of Bond Valence Parameters version 1999.3.26.

\section{References}

Aleksandrov, K. S. (1976). Kristallografiya, 21, 249-255. Aleksandrov, K. S. \& Bartolome, J. (2001). To be published. 
Alonso, J. A., Martínez-Lope, M. J., Casais, M. T., Aranda, M. A. G. \& Fernández-Díaz, M. T. (1999). J. Am. Chem. Soc. 121, 4754-4762.

Alonso, J. A., Martínez-Lope, M. J., Casais, M. T. \& Fernández-Díaz, M. T. (2000). Inorg. Chem. 39, 917-923.

Anan'eva, G. V., Ivanov, A. O., Merkulyaeva, T. I. \& Mochalov, I. V. (1978). Kristallografiya, 23, 200-202.

Bochu, B., Deschizeaux, M. N., Joubert, J. C., Collomb, A., Chenavas, J. \& Marezio, M. (1979). J. Solid State Chem. 29, 291-298.

Brese, N. E. \& O'Keeffe, M. (1991). Acta Cryst. B47, 192-197.

Brixner, L. H. (1960). J. Inorg. Nucl. Chem. 14, 225-230.

Brown, I. D. (1978). Chem. Soc. Rev. 7, 359-376.

Brown, I. D. (1992). Acta Cryst. B48, 553-572.

Brown, I. D. \& Altermatt, D. (1985). Acta Cryst. B41, 244-247.

Burns, G. \& Glazer, A. M. (1990). Space Groups for Solid State Scientists, 2nd ed., Appendix A9-6. San Diego: Academic Press.

Buttner, R. H. \& Maslen, E. N. (1988). Acta Cryst. C44, 1707-1709.

Carlson, S., Larsson, A. K. \& Rohrer, F. E. (2000). Acta Cryst. B56, 189-196.

Clearfield, A. (1963). Acta Cryst. 16, 135-142.

Colla, E. L., Reaney, I. M. \& Setter, N. (1993). J. Appl. Phys. 74, 3414 3425.

Darlington, C. N. W. \& Knight, K. S. (1999a). Physica B, 266, 368-372.

Darlington, C. N. W. \& Knight, K. S. (1999b). Acta Cryst. B55, 24 30.

Demazeau, G., Parent, C., Pouchard, M. \& Hagenmuller, P. (1972). Mater. Res. Bull. pp. 913-920.

Deschanvres, A., Raveau, B. \& Tollemer, M. F. (1967). Bull. Chem. Soc. Fr. pp. 4077-4078.

Dickens, P. G. \& Powell, A. (1991). J. Mater. Chem. 1, 137-138.

García-Muñoz, J. L., Rodríguez-Carvajal, J., Lacorre, P. \& Torrance, J. B. (1992). Phys. Rev. B, 46, 4414-4425.

Gingl, F., Vogt, T., Akiba, E. \& Yvon, K. (1999). J. Alloys Compd, 282, 125-129.

Glazer, A. M. (1972). Acta Cryst. B28, 3384-3392.

Glazer, A. M. (1975). Acta Cryst. A31, 756-762.

Goldschmidt, V. M. (1926). Naturwissenschaften, 14, 477-485.

Hinatsu, Y. \& Itoh, M. (1996). J. Solid State Chem. 132, 337-341.

Hohnstedt, C. \& Meyer, G. (1993). Z. Anorg. Allg. Chem. 619, 13741358.

Hönle, W., Miller, G. \& Simon, A. (1988). J. Solid State Chem. 75, 147155.

Howard, C. J. \& Kennedy, B. J. (1999). J. Phys. Condens. Matter, 11, 3229-3236.

Howard, C. J., Kennedy, B. J. \& Chakoumakos, B. C. (2000). J. Phys. Condens. Matter, 12, 349-365.

Howard, C. J., Knight, K. S., Kennedy, B. J. \& Kisi, E. H. (2000). J. Phys. Condens. Matter, 12, L677-L683.

Howard, C. J. \& Stokes, H.T. (1998). Acta Cryst. B54, 782-789.

Hutton, J. \& Nelmes, R. J. (1981). J. Phys. C Solid State Phys. 14, 1713-1736.

Hutton, J., Nelmes, R. J. \& Scheel, H. J. (1981). Acta Cryst. A37, 916920.

Hwang, H. Y., Palstra, T. T. M., Cheong, S.-W. \& Batlogg, B. (1995). Phys. Rev. B, 52, 15046-15049.

Ito, K., Tezuka, K. \& Hinatsu, Y. (2001). J. Solid State Chem. 157, 173 179.

Kennedy, B. J., Howard, C. J. \& Chakoumakos, B. C. (1999a). Phys. Rev. B, 59, 4023-4027.

Kennedy, B. J., Howard, C. J. \& Chakoumakos, B. C. (1999b). Phys. Rev. B, 60, 2972-2975.

Kennedy, B. J., Howard, C. J. \& Chakoumakos, B. C. (1999c). J. Phys. Condens. Matter, 11, 1479-1488.

Kennedy, B. J., Prodjosantoso, A. K. \& Howard, C. (1999). J. Phys. Condens. Matter, 11, 6319-6327.

Kijima, N., Tanaka, K. \& Marumo, F. (1981). Acta Cryst. B37, 545548.

Kijima, N., Tanaka, K. \& Marumo, F. (1983). Acta Cryst. B39, 557561.
Kobayashi, H., Nagata, M., Kanno, R. \& Kawamoto, Y. (1994). Mater. Res. Bull. 29, 1271-1280.

Labeau, M., Bochu, B., Joubert, J. C. \& Chenavas, J. (1980). J. Solid State Chem. 33, 257-261.

Leinenweber, K. \& Parise, J. (1995). J. Solid State Chem. 114, 277-281.

Liu, G., Zhao, X. \& Eick, H. A. (1992). J. Alloys Compd, 187, 145156.

Luaña, V., Costales, A. \& Pendás, A. M. (1997). Phys. Rev. B, 55, 4285-4297.

Malecki, G. \& Ratuszna, A. (1999). Powder Diffr. 14, 25-30.

Marchand, R., Laurent, Y., Guyader, J., L'Haridon, P. \& Verdier, P. (1991). J. Eur. Ceram. Soc. 8, 197-213.

Marezio, M., Dernier, P. D., Chenavas, J. \& Joubert, J. C. (1973). J. Solid State Chem. 6, 16-20.

Marezio, M., Remeka, J. P. \& Dernier, P. D. (1970). Acta Cryst. B26, 2008-2022.

Marti, W., Fischer, P., Altorfer, F., Scheel, H. J. \& Tadin, M. (1994). J. Phys. Condens. Matter, 6, 127-135.

Mehta, A., Berliner, R. \& Smith, R. W. (1997). J. Solid. State Chem. 130, 192-198.

Miyata, N., Tanaka, K. \& Marumo, F. (1983). Acta Cryst. B39, 561564

Newnham, R. E. (1997). MRS Bull. 22, 20-34.

Odenthal, R.-H. \& Hoppe, R. (1971). Monatsh. Chem. 102, 1340 1350.

O'Keeffe, M. \& Hyde, B. G. (1977). Acta Cryst. B33, 3802-3813.

Ozaki, Y., Ghedira, M., Chenavas, J., Joubert, J. C. \& Marezio, M. (1977). Acta Cryst. B33, 3615-3617.

Park, J.-H. \& Parise, J. B. (1997). Mater. Res. Bull. 32, 1617-1624.

Park, J.-H., Woodward, P. M. \& Parise, J. B. (1998). Chem. Mater. 10, 3092-3100.

Peschel, S., Ziegler, B., Schwarten, M. \& Babel, D. (2000). Z. Anorg. Allg. Chem. 626, 1561-1566.

Pickardt, J., Schendler, T. \& Kolm, M. (1988). Z. Anorg. Allg. Chem. 560, 153-157.

Ramirez, A. P., Subramanian, M. A., Gardel, M., Blumberg, G., Li, D., Vogt, T. \& Shapiro, S. M. (2000). Solid State Commun. 115, $217-$ 220.

Rao, G. H., Bärner, K. \& Brown, I. D. (1998). J. Phys. Condens. Matter, 10, L575-L763.

Rey, M. J., Dehaudt, P. H., Joubert, J. C., Lambert-Andron, B., Cyrot, M. \& Cyrot-Lackmann, F. (1990). J. Solid State. Chem. 86, 101-108.

Ross, N. L. (1998). Phys. Chem. Miner. 25, 597-602.

Roth, R. S. (1957). J. Res. Natl. Bur. Stand. 58, 75-88.

Salinas-Sanchez, A., Garcia-Munoz, J. L., Rodriguez-Carvajal, J., Saez-Puche, R. \& Martinez, J. L. (1992). J. Solid State Chem. 100, 201-211.

Sarkozy, R. F., Moeller, C. W. \& Chamberland, B. L. (1974). J. Solid State Chem. 9, 242-246.

Sasaki, S., Prewitt, C. T. \& Bass, J. D. (1987). Acta Cryst. C43, 1668 1674.

Shannon, R. D. (1976). Acta Cryst. A32, 751-767.

Shaplygin, I. S. \& Lazarev, V. B. (1976). Zh. Neorg. Khim. 21, 23262330.

Shimizu, Y., Syono, Y. \& Akimoto, S. (1970). High Temp. High Press. 2, 113-120.

Shishido, T., Nakagawa, S., Yoshikawa, A., Horiuchi, H., Tanaka, M., Sasaki, T. \& Fukuda, T. (1995). Nippon Kagaku Kaishi, 9, 697-702.

Sleight, A. W., Gillson, J. L. \& Bierstedt, P. E. (1975). Solid State Commun. 17, 27-28.

Smith, A. J. \& Welch, A. J. E. (1960). Acta Cryst. 13, 653-656.

Subramanian, M. A, Li, D., Duan, N., Reisner, B. A. \& Sleight, A. W. (2000). J. Solid State Chem. 151, 323-325.

Svensson, G. \& Werner, P.-E. (1990). Mater. Res. Bull. 25, 9-14

Thornton, G., Tofield, B. C. \& Hewat, A. W. (1986). J. Solid State Chem. 61, 301-307.

Töpfer, J. \& Goodenough, J. B. (1997). J. Solid State Chem. 130, 117128. 


\section{research papers}

Trolier-McKinstry, S. \& Newnham, R. E. (1993). MRS Bull. 18, 27-33. Vaselechko, L., Matkovskii, A., Savytskii, D., Suchocki, A. \& Wallrafen, F. (1999). J. Alloys Compd, 291, 57-65.

Vegas, A., Vallet-Regí, M., González-Calbet, J. M. \& Alario-Franco, M. A. (1986). Acta Cryst. B42, 167-172.

Woodward, P. M. (1997a). J. Appl. Cryst. 30, 206-207.

Woodward, P. M. (1997b). Acta Cryst. B53, 44-66.

Woodward, P. M. (1997c). Acta Cryst. B53, 32-43.
Yamamoto, A., Khasanova, N. R., Izumi, F., Wu, X.-J., Kamiyama, T., Torii, S. \& Tajima, S. (1999). Chem. Mater. 11, 747-753.

Zhao, C., Feng, S., Chao, Z., Shi, C., Xu, R. \& Ni, J. (1996). Chem. Commun. 14, 1641-1642.

Zhao, Y., Weidner, D. J., Parise, J. B. \& Cox, D. E. (1993). Phys. Earth Planet. Inter. 76, 1-16.

Zhurova, E. A., Zavodnik, V. E. \& Tsirel'son, V. G. (1995). Kristallografiya, 40, 816-823. 